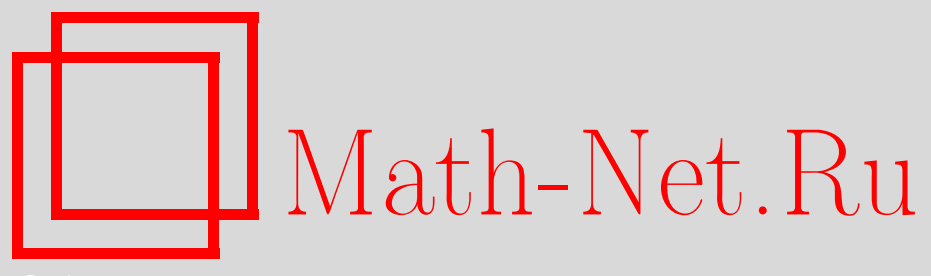

Ю. С. Ледяев, Д. С. Триман, Суб- и суперградиенты огибающих, полунепрерывных замыканий и пределов последовательностей функций, УМН, 2012, том 67, выпуск 2, 157-186

DOI: https://doi.org/10.4213/rm9466

Использование Общероссийского математического портала Math-Net.Ru подразумевает, что вы прочитали и согласны с пользовательским соглашением http: //www . mathnet.ru/rus/agreement

Параметры загрузки:

IP: 3.85 .73 .92

26 апреля 2023 г., 15:53:51

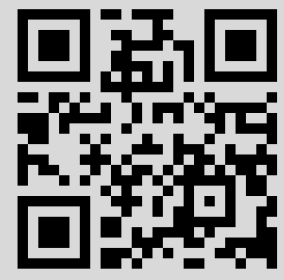




\title{
Суб- и суперградиенты огибающих, полунепрерывных замыканий и пределов последовательностей функций
}

\author{
Ю. С. Ледяев, Дж. С. Триман
}

Огибающие параметрических семейств функций

$$
\sup _{\gamma \in \Gamma} f_{\gamma}(x) \quad \text { или } \inf _{\gamma \in \Gamma} f_{\gamma}(x)
$$

представляют собой типичные недифференцируемые функции, возникающие в негладком анализе, теории оптимизации, теории управления, теории обобщенных решений уравнений первого порядка и других приложениях.

В этом обзоре мы получаем формулы для суб- и суперградиентов огибающих семейств полунепрерывных снизу функций, их полунепрерывных замыканий и пределов и Г-пределов последовательностей функций. Единый метод вывода этих формул для полунепрерывных функций основан на использовании многомерных формул конечных приращений для множеств и негладких функций.

Эти результаты используются для доказательства обобщений теорем Юнга и Хелли для многообразий неположительной кривизны, для доказательства единственности решений некоторых оптимизационных задач и нового доказательства известного вариационного принципа Стегалла для гладких банаховых пространств. Мы также получаем необходимые условия для точек $\varepsilon$-максимума полунепрерывных снизу функций.

Библиография: 47 названий.

Ключевые слова: нелинейный функциональный анализ, негладкий анализ, верхние и нижние огибающие, обобщение теорем Юнга, Хелли, Стегалла.

\section{СОДЕРЖАНИЕ}

1. Введение............................................ 158

2. Обобщенные формулы конечных приращений $\ldots \ldots \ldots \ldots \ldots \ldots \ldots \ldots \ldots$

3. Субградиенты верхней огибающей параметрических семейств функций 166

4. Субградиенты нижних огибающих параметрических семейств функций 173

4.1. Единственность точек минимума .................... 175

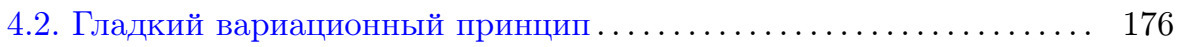

4.3. Ближайшие точки для замкнутых подмножеств гильбертова пространства .................................. 177

(C) Ю. С. ЛЕдяЕв, Дж.С. Триман, 2012 
5. Суперградиенты полунепрерывных сверху замыканий полунепрерывных снизу функций . . . . . . . . . . . . . . . . . . . . 178

6. Субградиенты пределов и эпи-пределов последовательностей функций 181 Список литературы . . . . . . . . . . . . . . . . . . . . . . . 184

\section{1. Введение}

Изучение инфинитезимальных (дифференциальных) свойств функции является основой анализа функций действительных переменных, который увязывает инфинитезимальные свойства функций с их глобальным поведением. Для полунепрерывных снизу функций, которые недифференцируемы в классическом смысле, подходящим для замены стандартных производных инфинитезимальным конструктом является субградиент. Теория негладкого анализа [10], [11], [17], которая развивалась на протяжении последних десятилетий, использует это фундаментальное понятие.

Чтобы объяснить понятие субградиента негладкой функции $f: \mathbb{X} \rightarrow \mathbb{R}$, рассмотрим две классические геометрические интерпретации производной $f^{\prime}(x)$. Традиционно в одномерном случае $\mathbb{X}=\mathbb{R}$ производная $f^{\prime}(x)$ дифференцируемой функции $f$ отождествляется с тангенсом угла наклона касательной линии к графику функции $f$. Другая интерпретация $f^{\prime}(x)$, которая справедлива в многомерном случае $\mathbb{X}=\mathbb{R}^{n}$ или даже в случае гильбертова пространства $\mathbb{X}=\mathbb{H}$, идентифицирует $\left(f^{\prime}(x),-1\right)$ как перпендикуляр, или проксимальный нормалъный вектор, к надграфику $f$

$$
\text { epi } f:=\{(x, \alpha) \in \mathbb{X} \times \mathbb{R}: f(x) \leqslant \alpha\}
$$

(напомним, что вектор $w \in \mathbb{H}$ называется проксимальной нормалью к множеству $S$ в точке $x \in S$, если для некоторой точки $z \notin S$ точка $x$ является ближайшей к $z$ точкой из $S$ и $w=\lambda(z-x)$ для некоторого $\lambda>0)$.

Понятие проксимального субградиента (см. [11], [17]) для гильбертова пространства основано на этой интерпретации.

Именно, рассмотрим функцию $f: \mathbb{X} \rightarrow(-\infty, \infty]$ и точку $x \in \operatorname{dom} f:=$ $\{y: f(y)<\infty\}$. Вектор $\zeta \in \mathbb{X}$ называется проксимальным субградиентом $f$ в $x$, если вектор $(\zeta,-1)$ есть проксимальная нормаль к ері $f$ в $(x, f(x))$. Эквивалентное аналитическое определение проксимального субградиента $\zeta$ требует существования постоянной $\sigma>0$ и окрестности $U$ точки $x$ таких, что выполняется следующее неравенство:

$$
f(y)-f(x) \geqslant\langle\zeta, y-x\rangle-\sigma\|y-x\|^{2} \quad \forall y \in U .
$$

Обозначим множество всех проксимальных субградиентов функции $f$ в $x$ через $\partial_{\mathrm{P}} f(x)$. Проксимальный субдифференииал $\partial_{\mathrm{P}} f$ может быть пуст в некоторой точке $x$; однако для любой полунепрерывной функции $f$ он непуст на всюду плотном подмножестве области определения $\operatorname{dom} f$, если пространство $\mathbb{X}$ гильбертово. Подробное изложение анализа проксимальных субградиентов можно найти в монографии [17].

Возвращаясь к неравенству (1) и рассматривая квадратичную функцию

$$
g(y):=\langle\zeta, y\rangle-\sigma\|y-x\|^{2},
$$


заметим, что это неравенство (1) означает, что функция $f-g$ достигает локального минимума в точке $x$ и проксимальный субградиент $\zeta$ совпадает с $g^{\prime}(x)$. Эта интерпретация напоминает другое понятие субградиента, которое имеет смысл для более общих, чем гильбертовы, банаховых пространств, для которых существуют гладкие липшицевы функции-бугорки ("bump functions"), а именно гладкие по Фреше липшицевы функции с ограниченным носителем. Такие пространства называются гладкими банаховыми пространствами. Примером гладкого банахова пространства является рефлексивное банахово пространство.

В случае такого гладкого банахова пространства можно определить субградиент Фреше функции $f$ в $x$ как линейный функционал $\zeta:=g^{\prime}(x)$ для гладкой по Фреше функции $g: \mathbb{X} \rightarrow \mathbb{R}$ такой, что функция $f-g$ достигает локального минимума в $x$. Конечно, в этом случае субградиент не имеет такой ясной геометрической интерпретации, как упомянутый выше проксимальный субградиент.

Мы используем обозначение $\partial_{\mathrm{F}} f$ для субдифференциала Фреше функции $f$, который состоит из всех таких субградиентов.

Хорошие описания анализа для таких субдифференциалов можно найти в [6], [27] и монографиях [7], [36], [37], [43].

Суперградиент $\zeta$ полунепрерывной сверху функции $f: \mathbb{X} \rightarrow(-\infty,+\infty]$ определяется как отрицательное значение субградиента функции $-f$; другими словами, суперградиентом функции $f$ в $x$ является вектор $\zeta=g^{\prime}(x)$ для некоторой гладкой по Фреше функции $g$ такой, что $f-g$ достигает локального максимума в $x$. Супердифференциалом $\partial^{\mathrm{F}} f(x)$ функции $f$ называется множество всех суперградиентов $f$ в точке $x$.

В этой статье мы изучаем инфинитезимальные свойства огибающих параметрических семейств функций в терминах их суб- и суперградиентов.

Рассмотрим параметрическое семейство функций $f_{\gamma}: \mathbb{X} \rightarrow(-\infty,+\infty]$, определенных на гладком банаховом пространстве $\mathbb{X}$, где параметр $\gamma$ принимает значения в некотором множестве $Г$.

Верхними и нижними огибающими этого семейства называются функции

$$
f(x):=\sup _{\gamma \in \Gamma} f_{\gamma}(x), \quad h(x):=\inf _{\gamma \in \Gamma} f_{\gamma}(x) .
$$

Подобные огибающие функции встречаются в многочисленных приложениях в теории оптимизации, теории управления и теории уравнений в частных производных (см., например, [17]). Также понятно, что такие огибающие функции не обязаны быть дифференцируемыми (или хотя бы непрерывными), даже если функции $f_{\gamma}$ являются гладкими.

В этой статье мы приводим формулы для представления субградиентов огибающей функции $f$ в $(2)$ в случае полунепрерывных снизу функций $f_{\gamma}$ в терминах субградиентов этих функций.

Мы также приводим формулы для суперградиентов полунепрерывного сверху замыкания

$$
\operatorname{usc} f(x):=\lim _{\delta \downarrow 0} \sup \{f(y):\|y-x\|<\delta\}
$$

полунепрерывной снизу функции $f$ и формулы для субградиентов полунепрерывного снизу замыкания нижней огибающей функции $h(2)$ для семейства 
полунепрерывных снизу функций $f_{\gamma}$,

$$
\operatorname{lsc} h(x):=\lim _{\delta \downarrow 0} \inf \{h(y):\|y-x\|<\delta\} .
$$

Также получены формулы для представления субградиентов пределов и эпипределов последовательностей полунепрерывных снизу функций $f_{i}(x)$ в терминах субградиентов $f_{i}$.

Подобные формулы для представления суперградиентов нижней огибающей $h$ в (2) в случае полунепрерывных сверху функций $f_{\gamma}$, субградиентов полунепрерывного снизу замыкания полунепрерывной сверху функции $f$ и полунепрерывного сверху замыкания верхней огибающей $f(2)$ семейства полунепрерывных сверху функций $f_{\gamma}$ легко получить из формул для субградиентов, используя упомянутую выше связь между суперградиентами и субградиентами.

Чтобы продемонстрировать характерный вид этих формул, напомним классические результаты по инфинитезимальным свойствам огибающих функций, полученные Дж. М. Данскином, [18], [19], Б. Н. Пшеничным [39], [40] и В. Ф. Демьяновым [23]-[25]. Они показали, что при некоторых условиях дифференцируемости $f_{\gamma}$ и регулярной зависимости производных от $\gamma \in \Gamma$ огибающие функции имеют производные по направлению

$$
D f(x ; v):=\lim _{t \downarrow 0} \frac{f(x+t v)-f(x)}{t},
$$

и нашли формулы для представления таких производных.

В частности, если предположить, что $Г$ - счетно компактное множество и функции $(x, \gamma) \mapsto f_{\gamma}(x),(x, \gamma) \mapsto f_{\gamma}^{\prime}(x)$ непрерывны на $\mathbb{X} \times \Gamma$, то

$$
D f(x ; v)=\max _{\gamma \in \Gamma(x)}\left\langle f_{\gamma}^{\prime}(x), v\right\rangle
$$

где $\Gamma(x)$ - множество максимизирующих значений $\gamma$ :

$$
\Gamma(x):=\left\{\gamma \in \Gamma: f_{\gamma}(x)=f(x)\right\}
$$

Заметим, что в этом случае функция $v \mapsto D f(x ; v)$ является опорной функцией [41]

$$
D f(x ; v)=\max _{\zeta \in M(x)}\langle\zeta, v\rangle
$$

выпуклого множества

$$
M(x)=\overline{\operatorname{co}}\left\{f_{\gamma}^{\prime}(x): \gamma \in \Gamma(x)\right\}
$$

где $\overline{\text { со }} A$ обозначает выпуклую оболочку множества $A$.

Это означает, что для конечного множества $\Gamma=\{1, \ldots, K\}$ и выпуклых гладких функций $f_{\gamma}$ субградиент (в смысле выпуклого анализа [9], [41]) верхней огибающей функции $f(2)$ имеет представление

$$
\zeta=\sum_{\gamma \in \Gamma(x)} \alpha_{\gamma} f_{\gamma}^{\prime}(x), \quad \text { где } \quad \alpha_{\gamma} \geqslant 0, \quad \sum_{\gamma \in \Gamma(x)} \alpha_{\gamma}=1 .
$$


В этой работе показывается, что при весьма общих предположениях субградиенты верхней огибающей (2) аппроксимируются выпуклыми комбинациями субградиентов полунепрерывных снизу функций $f_{\gamma}$, подобно тому как это происходит в гладком выпуклом случае (7). Общий выпуклый случай был рассмотрен в [45].

Настоящая статья основана на нашем препринте 2002 года, который содержал результаты по формулам для представлений суб- и суперградиентов огибающих функций, полунепрерывных замыканий и пределов последовательностей функций. Некоторые варианты результатов из этого препринта опубликованы в монографиях [7] и [43].

В этой статье, которая носит обзорный характер, наряду с развитием результатов из упомянутого препринта мы приводим некоторые их приложения, которые описаны ниже.

Изложение организовано по следующему плану. Раздел 2 содержит результаты по многомерным вариантам формулы конечных приращений Лагранжа для полунепрерывных снизу и непрерывных функций для случая гладких банаховых пространств.

Эти результаты систематически используются для доказательств основных теорем. Результаты о представлении субградиентов верхних огибающих семейств полунепрерывных снизу функций содержатся в разделе 3. Там же мы демонстрируем приложение этих результатов для вывода обобщений классических теорем Юнга и Хелли для многообразий неположительной кривизны.

Формулы для представления субградиентов нижних огибающих или их полунепрерывного замыкания приводятся в разделе 4. Здесь же мы приводим применения этих результатов для получения обобщения вариационного принципа Стегалла. Это обобщение получено для случая замкнутых ограниченных невыпуклых множеств и заменяет предположение о наличии свойства РадонаНикодима для пространства $\mathbb{X}$ предположением о гладкости его сопряженного пространства $\mathbb{X}^{*}$.

Мы также демонстрируем применение нижних огибающих и их инфинитезимальных свойств для получения условий существования и единственности минимумов в задачах оптимизации.

Раздел 5 содержит результаты по представлению суперградиентов полунепрерывных снизу функций и их применение для вывода необходимых условий максимума для таких функций. В заключительном разделе 6 мы получаем представление субградиентов пределов и эпи-пределов последовательностей полунепрерывных снизу функций.

В статье используются следующие обозначения: $\|x\|$ обозначает норму вектора $x$ в $\mathbb{X}, B$ - шар радиуса 1 с центром в нуле в пространстве $\mathbb{X},\langle\zeta, x\rangle$ обозначает действие линейного непрерывного функционала $\zeta \in \mathbb{X}^{*}$ на вектор $x \in \mathbb{X}, \mathbb{R}_{+}:=(0,+\infty)$.

Для функции $f$ и множества $Y$ величину $\inf _{y \in Y} f(y)$ обозначаем через $\inf f(Y)$.

Алгебраические операции с множествами в линейном пространстве определяются стандартным образом:

$$
A+B:=\{x+y: x \in A, y \in B\}, \quad \alpha A:=\{\alpha x: x \in A\} .
$$


Мы используем термин "выпуклье коэффициенты" для обозначения набора чисел $\left\{\alpha_{k}\right\}$ таких, что

$$
\alpha_{k} \geqslant 0, \quad k=1, \ldots, K, \quad \text { и } \quad \sum_{k=1}^{K} \alpha_{k}=1 .
$$
Выпуклой комбинацией элементов $\left\{x_{k}\right\}$ называется выражение $\sum_{k=1}^{K} \alpha_{k} x_{k}$ для
некоторых выпуклых коэффициентов $\left\{\alpha_{k}\right\}$.

\section{2. Обобщенные формулы конечных приращений}

В доказательствах основных результатов этой статьи используется обобщение формулы конечных приращений ${ }^{1}$.

Классическая формула конечных приращений в анализе является по сути одномерным результатом. Именно, для дифференцируемой функции $f$ и произвольных точек $x, y \in \mathbb{X}$ найдется точка $z$ в интервале $(x, y)$ такая, что

$$
f(y)-f(x)=\left\langle f^{\prime}(z), y-x\right\rangle .
$$

Многомерное обобщение формулы конечных приращений было предложено в [12]-[14] для общего случая полунепрерывных снизу функций и гильбертова пространства. Чтобы проиллюстрировать многомерность этого обобщения, сформулируем его для случая гладкой функции $f$, точки $x$ и замкнутого ограниченного выпуклого множества $Y$ : для произвольного числа $r<$ $\inf f(Y)-f(x)$ найдется точка $z \in[x, Y]$ такая, что

$$
r<\inf _{y \in Y}\left\langle f^{\prime}(z), y-x\right\rangle
$$

где интервал $[x, Y]$ определяется как выпуклая оболочка множества $Y \cup\{x\}$.

В статье мы используем обобщение многомерной формулы конечных приращений из [13] для случая гладких банаховых пространств (см. [47]).

ТеОрема 2.1. Пусть $\mathbb{X}$ - гладкое банахово пространство, $Y \subset \mathbb{X}$ замкнутое ограниченное выпуклое множество, функция $f: \mathbb{X} \rightarrow(-\infty,+\infty]$ полунепрерывна снизу и $x \in \operatorname{dom} f$. Предположим, что $f$ ограничена снизу на $[x, Y]+\varepsilon_{0} B$ для некоторого $\varepsilon_{0}>0$. Тогда для любого $\varepsilon>0$ и любого $r$, удовлетворяющего

$$
r<\sup _{\delta>0} \inf f(Y+\delta B)-f(x),
$$

найдутся точка $z \in[x, Y]+\varepsilon B$ и субградиент $\zeta \in \partial_{\mathrm{F}} f(z)$ такие, что

$$
\begin{aligned}
f(z) & <f(x)+\max \{0, r\}+\varepsilon, \\
r & <\min _{y \in Y}\langle\zeta, y-x\rangle .
\end{aligned}
$$

\footnotetext{
${ }^{1}$ В русской и французской литературе классическая формула конечных приращений связывается с именем Лагранжа, в английской литературе этот результат называется теоремой о среднем значении (mean-value theorem).
} 
Заметим, что в теореме 2.1 можно опустить предположение об ограниченности функции $f$ снизу, используя метод статьи [34].

Также заметим, что оценка (8) для промежуточной точки $z$ бывает важна для некоторых приложений. В этой статье нам понадобится одномерный вариант предыдущего результата с иной оценкой для точки $z$.

ТеОрема 2.2. Пусть $\mathbb{X}$ - гладкое банахово пространство и функиия $f$ : $\mathbb{X} \rightarrow \mathbb{R}$ непрерьвна. Тогда для любъх $y, x \in \operatorname{dom} f, \varepsilon>0$ и числа $r$, удовлетворяющего неравенству

$$
r<f(y)-f(x)
$$

найдутся точка $z \in[x, y]+\varepsilon B$ и субградиент $\zeta \in \partial_{\mathrm{F}} f(z)$ такие, что

$$
\begin{aligned}
f(z) & >f(y)-\max \{0, r\}-\varepsilon, \\
r & <\langle\zeta, y-x\rangle .
\end{aligned}
$$

ДокАЗАтЕльство. Поскольку $f$ непрерывна и интервал $[x, y]$ компактен, можно предположить, что существуют положительные постоянные $\rho$ и $L$ такие, что

$$
|f(z)|<L \quad \forall z \in V:=[x, y]+\rho B .
$$

Для гладкого банахова пространства $\mathbb{X}$ существует, как мы упоминали, $C^{1}$-гладкая липшицева функция-“бугорок" с ограниченным носителем. Используя эту функцию, легко построить функцию $g: X \rightarrow[0,+\infty)$ такую, что

$$
g(0)<\frac{1}{2} \quad \text { и } \quad g(z) \geqslant 2 \quad \forall z \notin B .
$$

Зафиксируем $r<f(y)-f(x)$ и $\bar{r} \in(r, f(y)-f(x))$. Для $k \in \mathbb{N}$ рассмотрим функцию $\varphi_{k}(t):=f_{k}(y(t))+\bar{r} t$ на $[0,1]$, где $y(t):=y+t(x-y)$ и

$$
f_{k}(u):=\inf _{z \in V}\left[f(z)+g^{k}(k(z-u))\right]
$$

Определим функцию

$$
\Phi_{k}(t, z):=f(z)+g^{k}(k(z-y(t)))
$$

тогда мы имеем представление

$$
\varphi_{k}(t)=\inf _{z \in V} \Phi_{k}(t, z)+\bar{r} t
$$

Очевидно, что

$$
f_{k}(u) \leqslant f(u)+g^{k}(0) \quad \forall u \in V .
$$

Отсюда и из (13) сразу следуют неравенства

$$
\varphi_{k}(0)<f(y)+2^{-k}, \quad \varphi_{k}(1)<f(x)+\bar{r}+2^{-k} .
$$

Лемма 2.1. Для всех достаточно больиих $k$ выполнено неравенство

$$
\varphi_{k}(1)<\varphi_{k}(0) .
$$


Ниже мы докажем, что

$$
\lim _{k \rightarrow \infty} \varphi_{k}(0)=f(y)
$$

тогда утверждение леммы будет следовать непосредственно из определения $\bar{r}$ и второго неравенства в (17).

Действительно, рассмотрим $y_{k} \in V$ такое, что

$$
f\left(y_{k}\right)+g^{k}\left(k\left(y_{k}-y\right)\right)<\varphi_{k}(0)+2^{-k} .
$$

Благодаря (17)

$$
g^{k}\left(k\left(y_{k}-y\right)\right)<M,
$$

где постоянная $M$ определяется так:

$$
M:=\max \{2,2 L+\bar{r}+1\}
$$

В соответствии с (13), это влечет $\left\|y_{k}-y\right\|<1 / k$. Таким образом, из предыдущих неравенств и (17) получаем

$$
f\left(y_{k}\right)-2^{-k}<\varphi_{k}(0)<f(y)+2^{-k} .
$$

Отсюда следует (19), что и завершает доказательство леммы.

Функция $\varphi_{k}$ удовлетворяет условию Липшица на $[0,1]$ и достигает своего максимума на $[0,1]$ в некоторой точке $t_{k}$. Отметим, что, вследствие $(18)$, для всех достаточно больших $k$ выполнено неравенство $t_{k}<1$.

Выберем теперь $t_{k}^{\prime} \in\left(t_{k}, 1\right)$ такое, что

$$
t_{k}^{\prime}-t_{k}<\frac{1}{M^{k}}
$$

а также $z_{k} \in V$ такое, что

$$
\Phi_{k}\left(t_{k}^{\prime}, z_{k}\right)<f_{k}\left(y\left(t_{k}^{\prime}\right)\right)+\left(t_{k}^{\prime}-t_{k}\right)^{2} .
$$

Из (14), (12) и определения (20) постоянной $M$ получаем

$$
g^{k}\left(k\left(z_{k}-y\left(t_{k}^{\prime}\right)\right)\right)<f\left(y\left(t_{k}^{\prime}\right)\right)+g^{k}(0)+1+L<M .
$$

Как и прежде, согласно свойствам $g^{k}$ это влечет

$$
\left\|z_{k}-y\left(t_{k}^{\prime}\right)\right\| \leqslant \frac{1}{k}<\rho
$$

для всех больших $k$. Это также означает, что множество $Y_{k}:=z_{k}+2\left(t_{k}^{\prime}-t_{k}\right) B$ лежит в $V$ для больших $k$.

Заметим, что

$$
\begin{aligned}
& \varphi_{k}\left(t_{k}^{\prime}\right)>\Phi_{k}\left(t_{k}^{\prime}, z_{k}\right)+\bar{r} t_{k}^{\prime}-\left(t_{k}^{\prime}-t_{k}\right)^{2}, \\
& \varphi_{k}\left(t_{k}\right) \leqslant \inf _{z \in V} \Phi_{k}\left(t_{k}, z\right)+\bar{r} t_{k} .
\end{aligned}
$$


Поскольку $\varphi_{k}\left(t_{k}\right)-\varphi_{k}\left(t_{k}^{\prime}\right) \geqslant 0$, следующее неравенство справедливо для всех больших $k$ :

$$
\bar{r}\left(t_{k}^{\prime}-t_{k}\right)-\left(t_{k}^{\prime}-t_{k}\right)^{2}<\inf _{z \in Y_{k}} \Phi\left(t_{k}, z\right)-\Phi\left(t_{k}^{\prime}, z_{k}\right) .
$$

Используя многомерное обобщение формулы конечных приращений (теорема 2.1), мы получаем существование точки $\left(\tilde{t}_{k}, \tilde{z}_{k}\right)$ и субградиента $\left(\xi_{t}, \xi_{z}\right) \in$ $\partial_{\mathrm{F}} \Phi\left(\tilde{t}_{k}, \tilde{z}_{k}\right)$ таких, что

$$
\begin{gathered}
\bar{r}\left(t_{k}^{\prime}-t_{k}\right)-\left(t_{k}^{\prime}-t_{k}\right)^{2}<\inf _{z \in z_{k}+\sqrt{t_{k}^{\prime}-t_{k}} B}\left\langle\xi_{z}, z-z_{k}\right\rangle+\xi_{t}\left(t_{k}-t_{k}^{\prime}\right), \\
\left|\tilde{t}_{k}-t_{k}\right|+\left\|\tilde{z}_{k}-z_{k}\right\|<3 \sqrt{t_{k}^{\prime}-t_{k}} .
\end{gathered}
$$

Из (24) следует, что

$$
r-\left(t_{k}^{\prime}-t_{k}\right)<\xi_{t} .
$$

Пусть $w_{k}:=k\left(\tilde{z}_{k}-y\left(\tilde{t}_{k}\right)\right)$. Очевидно, что $\xi_{t}=k^{2} g^{k-1}\left(w_{k}\right)\left\langle g^{\prime}\left(w_{k}\right), y-x\right\rangle$. Используя (24), оценки (22), (21) и липшицевость $g$, получаем

$$
\left\|\xi_{z}\right\|<O\left(\sqrt{t_{k}^{\prime}-t_{k}}\right) .
$$

Непосредственно из определения субградиента следует, что субградиент $\xi_{z}$ функции $\Phi_{k}$ может быть представлен как сумма субградиента $f$ и производной функции $z \mapsto g^{k}(k(z-y(t)))$, а именно, существует вектор $\zeta_{k} \in \partial_{\mathrm{F}} f\left(\tilde{z}_{k}\right)$ такой, что

$$
\xi_{z}=\zeta_{k}+k^{2} g^{k-1}\left(w_{k}\right) g^{\prime}\left(w_{k}\right) .
$$

Тогда из (26) получаем

$$
\bar{r}-\left(t_{k}^{\prime}-t_{k}\right)<\langle\zeta, y-x\rangle+\left\|\xi_{z}\right\|\|y-x\| .
$$

Из этого неравенства, оценок $(27),(21)$ и определения $\bar{r}$ следует, что для любого $\varepsilon>0$ существуют вектор $\zeta:=\zeta_{k}$ и точка $z:=\tilde{z}_{k}$, удовлетворяющие неравенству (11) для всех достаточно больших $k$. Нам осталось только доказать неравенство (10).

Без потери общности можно предположить, что $t_{k} \rightarrow t^{*}$, когда $k \rightarrow \infty$, для некоторого $t^{*} \in[0,1]$. Из $(23)$ и $(25)$ следует, что

$$
\lim _{k \rightarrow \infty}\left\|\tilde{z}_{k}-y\left(t^{*}\right)\right\|=0 .
$$

Но мы также имеем

$$
\varphi_{k}(0)<\varphi_{k}\left(t_{k}\right) \leqslant f\left(y\left(t_{k}\right)\right)+2^{-k}+\bar{r} t_{k} .
$$

Беря предел при $k \rightarrow \infty$ и используя непрерывность $f$ и соотношение (19), мы получаем

$$
f(y) \leqslant f\left(y\left(t^{*}\right)\right)+\bar{r} t^{*} .
$$

Тогда (10) следует из того, что $\tilde{z}_{k}$ сходится к $y\left(t^{*}\right)$ и что $\bar{r}$ можно выбрать произвольно близко к $r$. 
Следующий пример, предложенный Дж. Чжу, показывает, что предположение непрерывности функции $f$ является существенным.

ПримеР 2.1. Рассмотрим полунепрерывную снизу функцию

$$
f(x):= \begin{cases}-x, & \text { если } x \leqslant \frac{1}{2}, \\ 1-x, & \text { если } x>\frac{1}{2},\end{cases}
$$

и пусть $r=-1 / 8$. Тогда

$$
r<f(1)-f(0)=0 .
$$

Заметим, что $\partial_{\mathrm{F}} f(x)=\{-1\}$ для всех $x \neq 1 / 2$ и существует только одна точка, а именно $z=1 / 2$, такая, что для некоторого $\zeta \in \partial_{\mathrm{F}} f(z)$

$$
r<\zeta(1-0)
$$

Однако $f(z)<f(1)-\max \{r, 0\}-1 / 8$, что противоречит (10).

\section{3. Субградиенты верхней огибающей параметрических семейств функций}

В этом разделе мы рассматриваем параметрическое семейство полунепрерывных снизу функций $f_{\gamma}: \mathbb{X} \rightarrow(-\infty,+\infty]$ с параметром $\gamma \in \Gamma$. Здесь мы доказываем формулы для представления субградиентов верхней огибающей функции $f$ в $(2)$, которая очевидно является полунепрерывной снизу.

Множество

$$
\begin{aligned}
G_{\delta}(x):=\{(z, \gamma) \in \mathbb{X} \times \Gamma: z \in[x, y]+\delta B \\
\\
\text { для некоторого } \left.y \in x+\delta B, f_{\gamma}(y) \geqslant f(x)-\delta\right\}
\end{aligned}
$$

играет роль максимизирующего множества $\Gamma(x)(6)$, которое мы рассматривали в случае более регулярных $f_{\gamma}$ и $Г$.

В случае непрерывных функций $f_{\gamma}$ мы используем меньшее множество

$$
G_{\delta}^{\circ}(x):=\left\{(z, \gamma) \in \mathbb{X} \times \Gamma: z \in x+\delta B, f_{\gamma}(z) \geqslant f(x)-\delta\right\},
$$

чтобы характеризовать субградиенты $f$.

ПРЕДПОЛОЖЕНИЕ 3.1. Выполняется любое из следующих условий:

а) пространство $\mathbb{X}$ реблексивно;

b) для любого $x \in \mathbb{X}$ найдутся положительнье $L$ и $\delta$ такие, что для произвольных выпуклых коэффициентов $\left\{\alpha_{k}\right\}$, пар $\left(x_{k}, \gamma_{k}\right) \in G_{\delta}(x)$ и субградиентов $\zeta_{k} \in \partial_{\mathrm{F}} f_{\gamma_{k}}\left(x_{k}\right), k=1, \ldots, K$, выполнено неравенство

$$
\left\|\sum_{k=1}^{K} \alpha_{k} \zeta_{k}\right\| \leqslant L .
$$

Заметим, что условие b) выполняется, если функции $f_{\gamma}$ удовлетворяют условию Липшица равномерно по $\gamma$. 
Теорема 3.1. Пусть $\mathbb{X}$ - гладкое банахово пространство, функиии $f_{\gamma}$ : $\mathbb{X} \rightarrow(-\infty,+\infty]$ полунепрерывны снизу, выполняется предположение 3.1 u $\zeta \in \partial_{\mathrm{F}} f(x)$, где $f$ определена в (2). Тогда для любих $\varepsilon>0$ и $\delta>0$ существуют выпуклые коэффициенты $\left\{\alpha_{k}\right\}$ и пары $\left(x_{k}, \gamma_{k}\right) \in G_{\delta}(x), k=1, \ldots, K$, такие, yтo

$$
\zeta \in \sum_{k=1}^{K} \alpha_{k} \partial_{\mathrm{F}} f_{\gamma_{k}}\left(x_{k}\right)+\varepsilon B .
$$

Если функиии $f_{\gamma}$ непрерывны, то множество $G_{\delta}(x)$ может быть заменено менвшим множеством $G_{\delta}^{\circ}(x)$.

ДокАЗАтЕЛьСтво. Пусть $\zeta \in \partial_{\mathrm{F}} f(x)$, тогда для некоторой гладкой функции $g$ функция $f-g$ достигает локального минимума в $x$ и $\zeta=g^{\prime}(x)$. Зафиксируем произвольное достаточно малое $\delta>0$ и выберем произвольный вектор $v \in B$. Тогда для любого $t \in(0, \delta)$ выполнено неравенство

$$
f(x+t v)-f(x) \geqslant g(x+t v)-g(x) .
$$

Выберем $\gamma$ такое, что

$$
f_{\gamma}(x+t v)>f(x+t v)-t^{2} .
$$

Тогда из предыдущих соотношений следует, что

$$
f_{\gamma}(x+t v)-f_{\gamma}(x)>g(x+t v)-g(x)-t^{2} .
$$

Применяя одномерный вариант теоремы 2.1, где $r=g(x+t v)-g(x)-t^{2}$ и $Y:=\{y\}, y:=x+t v$, мы получаем существование точки $z$ вблизи $[x, x+t v]$ и субградиента $\xi \in \partial_{\mathrm{F}} f_{\gamma}(z)$ таких, что

$$
\langle\xi, x+t v-x\rangle>g(x+t v)-g(x)-t^{2} .
$$

Заметим, что $z$ может быть выбрана произвольно близко к $[x, y]$ и можно предположить, благодаря выбору $\gamma$, что для любого достаточно малого $t$

$$
(z, \gamma) \in G_{\delta}(x)
$$

В случае непрерывных функций $f_{\gamma}$ мы можем использовать одномерную формулу конечных приращений из теоремы 2.2 , чтобы показать, что $z$ удовлетворяет неравенству

$$
f_{\gamma}(z)>f_{\gamma}(y)-|g(x+t v)-g(x)|-t^{2}
$$

Поэтому в случае непрерывности функций $f_{\gamma}$ мы получаем в силу (30) и выбора $\gamma$, что для произвольного малого $t$

$$
(z, \gamma) \in G_{\delta}^{\circ}(x)
$$

Пусть $M$ есть замыкание выпуклого множества всех выпуклых комбинаций

$$
\sum_{k=1}^{K} \alpha_{k} \zeta_{k}
$$


где $\zeta_{k} \in \partial_{\mathrm{F}} f_{\gamma_{k}}\left(x_{k}\right)$ и $\left(x_{k}, \gamma_{k}\right) \in G_{\delta}(x)$ (множество $G_{\delta}(x)$ заменяется множеством $G_{\delta}^{\circ}(x)$ в случае, если функции $f_{\gamma}$ непрерывны).

Нетрудно видеть, что $M$ есть выпуклое замкнутое множество в $\mathbb{X}^{*}$ и из (29) следует, что для всех малых $t$

$$
\sup _{\xi \in M}\langle\xi, v\rangle>\frac{g(x+t v)-g(x)}{t}-t .
$$

Беря предел при $t \downarrow 0$ и замечая, что $v \in B$ произвольно, из (31) мы получаем

$$
\inf _{v \in B} \sup _{\xi \in M}\langle\xi-\zeta, v\rangle \geqslant 0
$$

В соответствии с предположением 3.1 множество $B$ слабо компактно (если $\mathbb{X}$ рефлексивно) или $M$ слабо* компактно (если выполнено условие b)), поэтому можно использовать несимметричную теорему о минимаксе (см., например, [7]), чтобы получить

$$
\inf _{\xi \in M}\|\xi-\zeta\| \leqslant 0
$$

что означает существование $\xi \in M$ такого, что $\zeta=\xi$. По определению множества $M$ это влечет утверждение теоремы 3.1. Теорема доказана.

Следующий пример демонстрирует, что условие непрерывности функций $f_{\gamma}$ существенно для того, чтобы в этой теореме множество $G_{\delta}(x)$ можно было заменить меньшим множеством $G_{\delta}^{\circ}(x)$.

ПримеР 3.1. Рассмотрим верхнюю огибающую $f$ двух полунепрерывных функций: $f_{1}(x) \equiv 0$ и

$$
f_{2}(x):= \begin{cases}|x|+1, & \text { если } x \neq 0, \\ -1, & \text { если } x=0 .\end{cases}
$$

Заметим, что $\partial_{\mathrm{F}} f_{1}(x)=\{0\}$ для всех $x$ и $\partial_{\mathrm{F}} f_{2}(x)=\{\operatorname{sign} x\}$ для всех $x \neq 0$. Очевидно, что $\zeta=2 \in \partial_{\mathrm{F}} f(0)$, но

$$
f_{2}(0) \leqslant f(0)-1 \text {. }
$$

Используя теорему 3.1 и теорему Каратеодори о представлении точек выпуклой оболочки множества в $\mathbb{R}^{n}$, мы получаем следующее утверждение.

СлеДСТвиЕ 3.1. Пусть $\mathbb{X}=\mathbb{R}^{n}$ и функции $x \mapsto f_{\gamma}(x)$ непрерывны. Тогда для любых $\varepsilon>0 u \delta>0$ существуют выпуклые коэфбициенты $\left\{\alpha_{k}\right\}$, пары $\left(x_{k}, \gamma_{k}\right) \in G_{\delta}^{\circ}(x)$ и субградиенты $\xi_{k} \in \partial_{\mathrm{F}} f_{\gamma_{k}}\left(x_{k}\right), k=1, \ldots, n+1$, такие, что

$$
\zeta \in \sum_{k=1}^{n+1} \alpha_{k} \xi_{k}+\varepsilon B .
$$

Если функиии $f_{\gamma}$ дифберениируемь, то предыдущее представление имеет вид

$$
\zeta \in \sum_{k=1}^{n+1} \alpha_{k} f_{\gamma_{k}}^{\prime}\left(x_{k}\right)+\varepsilon B .
$$


Предполагая компактность множества $Г$ и непрерывность функции $(x, \gamma) \mapsto$ $f_{\gamma}(x)$, мы получаем полунепрерывность сверху многозначного отображения $(x, \delta) \mapsto G_{\delta}^{\circ}(x)$ в точке $(x, 0)$. Поэтому имеет место следующее утверждение.

Следствие 3.2. Пусть $\mathbb{X}=\mathbb{R}^{n}$, функиии $(x, \gamma) \mapsto f_{\gamma}(x),(x, \gamma) \mapsto f_{\gamma}^{\prime}(x)$ непрерывны и множество Г компактно. Тогда, если $\zeta \in \partial_{\mathrm{F}} f(x)$, то

$$
\zeta=\sum_{k=1}^{n+1} \alpha_{k} f_{\gamma_{k}}^{\prime}(x)
$$

для некоторых выпуклых коэффициентов $\left\{\alpha_{k}\right\}$ и некоторых $\gamma_{k} \in \Gamma(x)$.

Теоремы Хелли и Юнга для римановых многообразий неположительной кривизны. В этом пункте мы демонстрируем применение полученных результатов к доказательству вариантов классических теорем Хелли и Юнга [20] для односвязных многообразий неположительной кривизны (иногда называемых многообразиями Адамара-Картана [2]).

Напомним, что классическая теорема Хелли утверждает, что для набора замкнутых выпуклых множеств $S_{\gamma} \subset \mathbb{R}^{n}, \gamma \in \Gamma$, таких, что хотя бы одно из них компактно, мы имеем

$$
\bigcap_{\gamma \in \Gamma} S_{\gamma} \neq \varnothing
$$

если пересечение любых $n+1$ множеств из этого набора непусто.

Теорема Юнга для замкнутого ограниченного множества $S \subset \mathbb{R}^{n}$ устанавливает, что диаметр множества

$$
D(S):=\sup \left\{\left\|y_{1}-y_{2}\right\|: y_{1}, y_{2} \in S\right\}
$$

и радиус описанного шара

$$
R(S):=\inf \left\{r>0: S \subset x_{0}+r B\right\}
$$

удовлетворяют неравенству

$$
R(S) \leqslant \frac{1}{2} \sqrt{\frac{2 n}{n+1}} D(S) .
$$

Рассмотрим $n$-мерное гладкое риманово многообразие Адамара-Картана $M$. Напомним, что риманова метрика на касательном расслоении $T M$ определяет двойственность $\langle\xi, v\rangle$ между касательным вектором $v \in T_{x}(M)$ и кокасательным вектором $\xi \in T_{x}^{*}(M)$, а также нормы векторов $\|v\|,\|\xi\|$.

Известно, что для любых двух точек $x_{0}, x_{1}$ такого многообразия существует единственная кратчайшая геодезическая $c(s), s \in[0,1]$, соединяющая их. Мы используем каноническую параметризацию $c$, полагая $\left\|c^{\prime}(s)\right\|$ постоянной на $[0,1]$.

Определим касательный вектор $v\left(x_{0}, x_{1}\right):=c^{\prime}(0)$ и заметим, что $x_{0} \mapsto v\left(x_{0}, x\right)$ есть непрерывное векторное поле на $M$.

Легко видеть, что расстояние между точками $x_{0}$ и $x_{1}$ определяется следующим образом

$$
\rho\left(x_{0}, x_{1}\right):=\int_{0}^{1}\left\|c^{\prime}(s)\right\| d s=\left\|v\left(x_{0}, x_{1}\right)\right\| .
$$


Заметим, что для двух геодезических $c_{1}(s), c_{2}(s)$ функция $s \mapsto \rho\left(c_{1}(s), c_{2}(s)\right)$ выпукла.

Множество $S \subset M$ называется выпуклым, если для произвольных точек $x_{0}, x_{1}$ в $S$ геодезическая, соединяющая их, лежит в $S$. Функция $f: M \rightarrow \mathbb{R}$ называется выпуклой, если для любой геодезической $c(s)$ функция $s \mapsto f(c(s))$ выпукла.

Нетрудно проверить, что функция расстояния $x \mapsto d_{S}(x)$ до выпуклого множества $S$,

$$
d_{S}(x):=\inf _{y \in S}(x, y)
$$

является выпуклой.

Отметим, что функция расстояния дифференцируема в точке $x \notin S$. Тем не менее верхняя огибающая семейства таких функций уже является негладкой и анализ ее инфинитезимальных свойств требует развития соответствующих средств.

Такой негладкий анализ на гладких многообразиях был разработан в [35]. Субградиент полунепрерывной снизу функции $f: M \rightarrow(-\infty,+\infty]$ определяется аналогичным линейному пространству образом: вектор $\zeta \in T_{x}^{*}(M)$ называется субградиентом функции $f$ в $x$, если существует гладкая функция $g: M \rightarrow \mathbb{R}$ такая, что функция $f-g$ достигает локального минимума в $x$ и $\zeta=d g(x)$. Совокупность всех субградиентов функции $f$ в точке $x$ образует субдифференциал $\partial_{\mathrm{F}} f(x)$.

Тогда мы получаем очевидное необходимое условие локального минимума: если точка $x_{0}$ есть точка локального минимума, то

$$
0 \in \partial_{\mathrm{F}} f\left(x_{0}\right) .
$$

Мы имеем следующий аналог следствия 3.1 для субградиента верхней огибающей (2) (см. [32], [35]).

Зафиксируем произвольное выпуклое компактное (в слабой* топологии) семейство непрерывных векторных полей $W$ на $M$. Пусть $\zeta \in \partial_{\mathrm{F}} f(x)$, тогда для любой окрестности $U$ точки $x$ и произвольных $\delta>0$ и $\varepsilon>0$ существуют выпуклые коэффициенты $\left\{\alpha_{k}\right\}_{k=1}^{n+1}$, пары $\left(y_{k}, \gamma_{k}\right) \in G_{\delta, U}(x)$ и субградиенты $\xi_{k} \in \partial_{\mathrm{F}} f_{\gamma_{k}}\left(y_{k}\right), k=1, \ldots, n+1$, такие, что

$$
\left|\langle\zeta, v\rangle-\sum_{k=1}^{n+1} \alpha_{k}\left\langle\xi_{k}, v\right\rangle\right|<\varepsilon \quad \forall v \in W,
$$

где

$$
G_{\delta, U}(x):=\left\{(y, \gamma) \in U \times \Gamma: f_{\gamma}(y) \geqslant f(x)-\delta\right\} .
$$

Заметим, что необходимость использования векторного поля $v$ в (36) обусловлена тем, что на многообразии нельзя непосредственно сравнивать кокасательные векторы $d g(x)$ и $d g(y)$ в разных точках $x, y \in M$. Но можно сравнить действие этих векторов на векторное поле:

$$
\langle d g(x), v(x)\rangle \quad \text { и }\langle d g(y), v(y)\rangle .
$$

Мы используем характеризацию (36) субградиента верхней огибающей для доказательства обобщений теорем Хелли и Юнга для многообразий. 
В работе [32] был доказан несколько более общий вариант следующей теоремы Хелли для многообразий.

ТЕОРема 3.2. Пусть $M$ является гладким односвязным римановым многообразием неположительной кривизны и либо семейство $S_{\gamma}, \gamma \in \Gamma$, выпуклых замкнутых множеств конечно, либо хотя бы одно из этих множеств компактно. Если для любых $n+1$ множеств их пересечение непусто, то и пересечение всех множеств также непусто.

ДокАЗАтельство. Для доказательства теоремы рассмотрим верхнюю огибающую (см. (2)) семейства функций

$$
f_{\gamma}(x):=d_{S_{\gamma}}(x), \quad \gamma \in \Gamma .
$$

Нетрудно видеть, что существование точки $x_{0}$ такой, что

$$
f\left(x_{0}\right) \leqslant 0
$$

влечет за собой (32).

Предположим обратное - что для некоторых $x_{0} \in S$ и $r>0$

$$
\min _{x \in S} f(x)=f\left(x_{0}\right)>r,
$$

где $S$ - выпуклое компактное множество, содержащее все множества $S_{\gamma}$ (без ограничения общности можно предположить, что такое множество существует). Диаметр множества $S$ обозначим $d$.

Заметим, что точка $x_{0}$ есть точка минимума на $S$ функции $f$, и поскольку эта функция удовлетворяет на $M$ условию Липшица с постоянной 1 , то, используя штрафную функцию $d_{S}(x)$, можно показать (см. [11]), что $x_{0}$ есть также точка минимума функции

$$
f(x)+2 d_{S}(x)
$$

на всем многообразии $M$. Анализируя условия оптимальности функции (39) и демонстрируя, что (38) приводит к противоречию, мы получим, что выполняется (37), и теорема будет доказана.

Поскольку $x_{0}$ есть точка минимума функции (39), получаем, что 0 есть субградиент этой функции. Тогда из (36) следует, что для выпуклого семейства $W$ векторных полей, порожденного полями $v(x):=v\left(x, x_{1}\right)$ с $x_{1} \in S$, для произвольных $\varepsilon>0$ и $\delta>0$ найдутся выпуклые коэффициенты $\left\{\alpha_{k}\right\}_{k=1}^{n+1}$, пары $\left(y_{k}, \gamma_{k}\right) \in G_{\delta, U}(x)$ и субградиенты $\xi_{k} \in \partial_{\mathrm{F}}\left(f_{\gamma_{k}}+2 d_{S}\right)\left(y_{k}\right), k=1, \ldots, n+1$, такие, что

$$
\left|\sum_{k=1}^{n+1} \alpha_{k}\left\langle\xi_{k}, v\right\rangle\right|<\varepsilon \quad \forall v \in W .
$$

Можно полагать, что $f\left(y_{k}\right)>r$; рассмотрим общую точку $x_{1}$ множеств $S_{\gamma_{k}}$, $k=1, \ldots, n+1$, которая существует согласно предположению теоремы 3.2 . Это означает, что

$$
f_{\gamma_{k}}\left(x_{1}\right)=0
$$


Пусть $c\left(s ; x, x_{1}\right)$ есть геодезическая, соединяющая точки $x$ и $x_{1}$, тогда векторное поле $v(x)=c^{\prime}\left(0 ; x, x_{1}\right)$ принадлежит $W$ и

$$
\left\langle\xi_{k}, v\right\rangle=\left.\frac{d}{d s} g\left(c\left(s ; y_{k}, x_{1}\right)\right)\right|_{s=0}
$$

для некоторой гладкой функции $g$ такой, что функция $f_{\gamma_{k}}(x)+2 d_{S}(x)-g(x)$ достигает локального минимума в точке $y_{k}$. Отсюда следует, что

$$
\begin{aligned}
\left\langle\xi_{k}, v\right\rangle & \leqslant \liminf _{s \downarrow 0} \frac{f_{\gamma_{k}}\left(c_{k}(s)\right)-f_{\gamma_{k}}\left(c_{k}(0)\right)}{s} \\
& \leqslant \liminf _{s \downarrow 0} \frac{(1-s) f_{\gamma_{k}}\left(c_{k}(0)\right)+s f_{\gamma_{k}}\left(c_{k}(1)\right)-f_{\gamma_{k}}\left(c_{k}(0)\right)}{s}
\end{aligned}
$$

где $c_{k}(s):=c\left(s ; y_{k}, x_{1}\right)$ и последнее неравенство следует из выпуклости функции $s \mapsto f_{\gamma_{k}}\left(c_{k}(s)\right)$.

Таким образом, для каждого $r=1, \ldots, n+1$

$$
\left\langle\xi_{k}, v\right\rangle \leqslant f_{\gamma_{k}}\left(x_{1}\right)-f_{\gamma_{k}}\left(y_{k}\right)<-r .
$$

Это противоречит (40) для достаточно малого $\varepsilon$, что влечет равенство $f\left(x_{0}\right)=0$ и означает, что $x_{0}$ есть общая точка всех множеств $S_{\gamma}$, что доказывает теорему Хелли на $M$. Теорема доказана.

Теперь мы рассмотрим ограниченное множество $S \subset M$, определим его диаметр $D(S):=\sup \left\{\rho\left(y_{1}, y_{2}\right): y_{1}, y_{2} \in S\right\}$ и радиус описанного шара $R(S):=$ $\min \{f(x): x \in M\}$, где

$$
f(x):=\max \{\rho(x, y): y \in S\}
$$

Более общий вариант теоремы Юнга для многообразия ограниченной сверху кривизны можно найти в [22], здесь же мы доказываем эту теорему для многообразия неположительной кривизны.

ТеОрема 3.3. Пусть $M$ является $n$-мерным гладким односвязным римановым многообразием неположительной кривизны и множество $S \subset M$ ограничено. Тогда диаметр этого множества и радиус описанного шара удовлетворяют соотношению (33).

ДокАЗАТЕЛЬСтво. Без ограничения общности можно полагать, что $S$ замкнуто. Будем вместо $D(S)$ и $R(S)$ писать просто как $D$ и $R$.

Рассмотрим точку минимума $x_{0}$ функции $f$, определенной в $(41)$; тогда выполняется условие оптимальности (35). Функция $f(41)$ есть верхняя огибающая параметрического семейства гладких функций $x \mapsto \rho(x, y), y \in S$.

Из следствия 3.2 получаем, что найдутся выпуклые коэффициенты $\left\{\alpha_{k}\right\}$ и точки $y_{k}, k=1, \ldots, n+1$, такие, что субградиент 0 функции $f$ в $x_{0}$ может быть представлен в виде

$$
0=\sum_{k=1}^{n+1} \alpha_{k} d \rho\left(x_{0}, y_{k}\right)
$$


Заметим, что $\rho\left(x_{0}, y_{k}\right)=R, d \rho\left(x_{0}, y_{k}\right) \in T_{x_{0}}^{*}(M)$. При этом предыдущее равенство можно переписать в виде

$$
0=\sum_{k=1}^{n+1} \alpha_{k} v_{k}
$$

где

$$
v_{k}:=\frac{c^{\prime}\left(0 ; x_{0}, y_{k}\right)}{\left\|c^{\prime}\left(0 ; x_{0}, y_{k}\right)\right\|} \in T_{x_{0}}(M) .
$$

Для произвольного индекса $i$ получаем из (42) и условия выпуклости коэффициентов $\alpha_{k}$, что

$$
0=\sum_{k=1}^{n+1} \alpha_{k}\left\langle v_{i}, v_{k}\right\rangle, \quad\left\langle v_{i}, v_{i}\right\rangle=\sum_{k=1}^{n+1} \alpha_{k}\left\langle v_{i}, v_{i}\right\rangle
$$

Вычитая эти равенства друг из друга, получим

$$
1=\sum_{k \neq i} \alpha_{k}\left(1-\cos \beta_{k i}\right)
$$

где $\beta_{k i}$ - угол при вершине $x_{0}$ в геодезическом треугольнике с вершинами в $x_{0}, y_{k}$ и $y_{i}$.

Теперь мы воспользуемся теоремой косинуса для таких треугольников на многообразии неположительной кривизны [2]: для треугольника со сторонами $a, b$ и $c$ и углом $\beta$ между сторонами $a$ и $b$

$$
a^{2}+b^{2}-2 a b \cos \beta \leqslant c^{2}
$$

Для треугольника с вершинами в $x_{0}, y_{k}$ и $y_{i}$

$$
2 R^{2}\left(1-\cos \beta_{k i}\right) \leqslant D^{2}
$$

Отсюда и из (43) получаем $1 \leqslant \sum_{k \neq i} \alpha_{k} \frac{D^{2}}{2 R^{2}}$. Суммируя эти неравенства по $i$, получим $n+1 \leqslant n \frac{D^{2}}{2 R^{2}}$, что и завершает доказательство теоремы.

\section{4. Субградиенты нижних огибающих параметрических семейств функций}

Рассмотрим нижнюю огибающую $h(2)$ параметрического семейства полунепрерывных снизу функций $f_{\gamma}: \mathbb{X} \rightarrow(-\infty,+\infty], \gamma \in \Gamma$. Мы получим формулы для представления субградиентов нижней огибающей $h$ и ее полунепрерывного снизу замыкания $\tilde{h}:=\mathrm{lsc} h$. Отличительной чертой этого результата является то, что можно связать субградиент $h$ или $\tilde{h}$ и произволъный $\varepsilon$-минимум $\gamma$.

Вариант следующей теоремы 4.1 , в которой субградиент $\tilde{h}$ связывался с некоmорым $\varepsilon$-минимумом $\gamma$, был получен в [33] и использовался для вывода общих 
теорем о неявных многозначных функциях. Теорема 4.1 имеет отношение к результатам о стабильности в [26], [27] и к результатам работы [44]. Также оказалось, что она может быть использована для изучения вопросов существования и единственности минимумов для некоторых оптимизационных задач. В этом отношении она позволяет лучше понять результаты И. Экланда и Ж. Лебурга [29], [30].

Мы также используем эту теорему для вывода в п. 4.2 гладкого вариационного принципа, напоминающего хорошо известный вариационный принцип Стегалла [47].

Tеорема 4.1. Пусть $\mathbb{X}$ - гладкое банахово пространство, функиии $f_{\gamma}: \mathbb{X} \rightarrow$ $(-\infty,+\infty], \gamma \in \Gamma$, полунепрерьвны снизу и $\zeta \in \partial_{\mathrm{F}} \tilde{h}(x)$ (соответственно $\zeta \in$ $\left.\partial_{\mathrm{F}} h(x)\right)$. Тогда существует функиия $\varphi: \mathbb{R}_{+} \rightarrow \mathbb{R}_{+}$такая, что $\lim _{\varepsilon \rightarrow+0} \varphi(\varepsilon)=0$ и для любого малого $\varepsilon>0$ и любых $\left(\gamma_{\varepsilon}, x_{\varepsilon}\right)$, удовлетворяющих

$$
x_{\varepsilon} \in x+\varepsilon B \quad u \quad f_{\gamma_{\varepsilon}}\left(x_{\varepsilon}\right)<\tilde{h}(x)+\varepsilon \quad\left(\text { соответственно } f_{\gamma_{\varepsilon}}(x)<h(x)+\varepsilon\right),
$$

найдется $z \in x+2 \sqrt{\varepsilon} B$ такое, что

$$
\begin{aligned}
& f_{\gamma_{\varepsilon}}(z)<\tilde{h}(x)+O(\sqrt{\varepsilon})\left(\text { соответственно } f_{\gamma_{\varepsilon}}(z)<h(x)+O(\sqrt{\varepsilon})\right), \\
& \zeta \in \partial_{\mathrm{F}} f_{\gamma_{\varepsilon}}(z)+\varphi(\varepsilon) B .
\end{aligned}
$$

ДокАЗАтельство. Рассмотрим случай $\zeta \in \partial_{\mathrm{F}} \tilde{h}$. Из определения субградиента функции $\tilde{h}$ в $x$ следует, что для некоторой гладкой функции $g$ функция $\tilde{h}-g$ достигает локального минимума в $x$ и $\zeta=g^{\prime}(x)$. Зафиксируем малое $\sigma>0$ такое, что для любого $y \in x+2 \sigma B$

$$
\tilde{h}(y)-g(y) \geqslant \tilde{h}(x)-g(x) .
$$

Выберем $\varepsilon>0$ и произвольную пару $\left(x_{\varepsilon}, \gamma_{\varepsilon}\right)$, удовлетворяющую (44). Поскольку $f_{\gamma}(y) \geqslant \tilde{h}(y)$ для любых $y$ и $\gamma$, то, благодаря определению $\left(\gamma_{\varepsilon}, x_{\varepsilon}\right)$ и локальной липшицевости $g$, получаем из предыдущего неравенства

$$
\left(f_{\gamma_{\varepsilon}}(y)-g(y)\right)-\left(f_{\gamma_{\varepsilon}}\left(x_{\varepsilon}\right)-g\left(x_{\varepsilon}\right)\right)>-L \varepsilon \quad \forall y \in x_{\varepsilon}+2 \sigma B,
$$

где мы предположили, что $\varepsilon$ настолько мало, что $\left\|g^{\prime}(z)\right\| \leqslant L-1$ для всех $z \in x+\varepsilon B$ и некоторой постоянной $L$.

Применяя обобщенную формулу конечных приращений (теорема 2.1) для множества $Y:=x_{\varepsilon}+\sigma B$, точки $x_{\varepsilon}$ и $r=-L \varepsilon$, мы получаем существование точки $z \in x_{\varepsilon}+2 \sigma B$ и субградиента $\xi \in \partial_{\mathrm{F}} f_{\gamma_{\varepsilon}}(z)$ таких, что

$$
f_{\gamma_{\varepsilon}}(z)<f_{\gamma_{\varepsilon}}\left(x_{\varepsilon}\right)+g(z)-g\left(x_{\varepsilon}\right)+\varepsilon
$$

И

$$
-L \varepsilon \leqslant\left\langle\xi-g^{\prime}(z), y-x_{\varepsilon}\right\rangle \quad \forall y \in Y .
$$

Последнее неравенство означает, что

$$
\left\|\xi-g^{\prime}(z)\right\| \leqslant \frac{L \varepsilon}{\sigma} .
$$


Мы также имеем $\|z-x\|<\varepsilon+2 \sigma$. Предполагая, что $\varepsilon>0$ достаточно мало, можно положить $\sigma=\sqrt{\varepsilon} / 2$ и получить из предыдущих неравенств включение $(45)$ с

$$
\varphi(\varepsilon):=2 L \sqrt{\varepsilon}+\sup \left\{\left\|g^{\prime}(z)-g^{\prime}(x)\right\|:\|z-x\|<\varepsilon+\sqrt{\varepsilon}\right\} .
$$

Кроме того, $\varepsilon<\sqrt{\varepsilon}$ для малых $\varepsilon>0$.

Это дает оценку для положения $z$. Неравенство для $f_{\gamma_{\varepsilon}}(z)$ следует из $(46)$ и определения $x_{\varepsilon}$.

Заметим, что если $\zeta \in \partial_{\mathrm{F}} h(x)$, то функция $h$ полунепрерывна снизу в $x$ и $\tilde{h}(x)=h(x)$. Доказательство формулы в этом случае следует предыдущему доказательству с $x_{\varepsilon}=x$. Это обеспечивает те же самые неравенства с $L=1$. Теорема доказана.

Результат работы [33] частично содержится в следующем утверждении, которое вытекает непосредственно из теоремы 4.1.

СледСтвиЕ 4.1. Пусть $\zeta \in \partial_{\mathrm{F}} \tilde{h}(x)$ (соответственно $\left.\zeta \in \partial_{\mathrm{F}} h(x)\right)$. Тогда для любого $\varepsilon>0$ найдутся $(\gamma, z) \in \Gamma \times(x+\varepsilon B)$ такие, что

$$
f_{\gamma}(z)<\tilde{h}(x)+\varepsilon \quad\left(\text { соответственно } f_{\gamma}(z)<h(x)+\varepsilon\right)
$$

$u$

$$
\zeta \in \partial_{\mathrm{F}} f_{\gamma}(z)+\varepsilon B .
$$

4.1. Единственность точек минимума. Чтобы объяснить, как теорема 4.1 может быть применена к задаче единственности точки минимума, рассмотрим случай $C^{1}$-гладких функций $f_{\gamma}$ в предположении, что множество $\Gamma(x)$ параметров $\gamma$ таких, что

$$
f_{\gamma}(x)=h(x),
$$

непусто. А именно, используя прямое рассуждение, мы устанавливаем, что в этом случае субдифференциал $\partial_{\mathrm{F}} h(x)$ состоит из одного элемента. Очевидный пример показывает, что это не так без предположения дифференцируемости $f_{\gamma}$.

Теорема 4.2. Пусть $f_{\gamma}: \mathbb{X} \rightarrow \mathbb{R}$ дифферениируема в $x \in \mathbb{X}$ для любого $\gamma \in \Gamma(x), \Gamma(x)$ непусто и $\zeta \in \partial_{\mathrm{F}} h(x)$. Тогда субдифберенииал $\partial_{\mathrm{F}} h(x)$ состоит из одного элемента и

$$
\zeta=f_{\gamma}^{\prime}(x) \quad \forall \gamma \in \Gamma(x)
$$

Если, в дополнение, производные $f_{\gamma}^{\prime}(x)$ отличны друг от друга для всех $\gamma \in \Gamma$ то множество Г $(x)$ состоит из единственной точки минимума.

ДокАЗАТЕЛЬство. Пусть $\zeta=g^{\prime}(x)$, где $C^{1}$ - гладкая функция $g$ такова, что функция $h-g$ достигает минимума в $x$. Зафиксируем $\gamma \in \Gamma(x)$, тогда функция $f_{\gamma}-g$ также достигает минимума в точке $x$. Тогда $\left(f_{\gamma}-g\right)^{\prime}(x)=0$, что влечет

$$
\zeta=f_{\gamma}^{\prime}(x) \quad \forall \gamma \in \Gamma(x) .
$$

Это означает, что $\partial_{\mathrm{F}} h(x)$ состоит из одного элемента $\zeta$. Чтобы закончить доказательство, заметим, что если все производные $f_{\gamma}^{\prime}(x)$ различны для разных $\gamma \in \Gamma(x)$, то существует единственное $\gamma \in \Gamma(x)$ такое, что $\zeta=f_{\gamma}^{\prime}(x)$. Это означает, что множество точек минимума $\Gamma(x)$ состоит из единственной точки. Теорема доказана. 
Следующие два пункта демонстрируют примеры применения теоремы 4.1 для получения существования и единственности точек минимума в общем положении для вариационных принципов и ближайших точек к замкнутому множеству в гильбертовом пространстве.

Можно также использовать аналогичный подход для доказательства существования и единственности оптимальных управления в невыпуклых задачах оптимального управления [31].

4.2. Гладкий вариационный принцип. Выше мы применили теорему 4.1, чтобы установить единственность точки минимума для некоторых оптимизационных задач. Здесь мы используем эту теорему, чтобы доказать существование таких точек минимума для некоторых произвольно малых линейных возмущений исходной оптимизационной задачи. Именно, мы доказываем вариант гладкого вариационного принципа Стегалла [46] в предположении, что двойственное пространство $\mathbb{X}^{*}$ является гладким. Детальное введение в вариационные принципы можно найти в книге Р.Р. Фелпса [38] и работах [6], [28], [27]. В [16] можно найти похожий подход к выводу вариационного принципа Стегалла в гильбертовом пространстве с использованием проксимального анализа.

ТеОрема 4.3. Пусть $\mathbb{X}$ - банахово пространство и $\mathbb{X}^{*}$ - гладкое банахово пространство, функиия $f: \mathbb{X} \rightarrow(-\infty,+\infty]$ полунепрерывна и ограничена снизу, $S$ - замкнутое подмножество $\mathbb{X}$. Рассмотрим функцию

$$
h\left(x^{*}\right):=\inf \left\{f(y)+\left\langle x^{*}, y\right\rangle: y \in S\right\}
$$

и предположим, что $\zeta \in \partial_{\mathrm{F}} h\left(x^{*}\right)$ для некоторого $x^{*}$. Тогда существует единственная точка $y_{0} \in S$ минимума функции

$$
y \mapsto f(y)+\left\langle x^{*}, y\right\rangle
$$

на $S$.

ДокАЗАТЕЛЬСтво. Применяя теорему 4.1 к нижней огибающей (47), получим, что для любого $\varepsilon>0$ и любого $y_{\varepsilon}$ такого, что

$$
f\left(y_{\varepsilon}\right)+\left\langle x^{*}, y_{\varepsilon}\right\rangle<h\left(x^{*}\right)+\varepsilon,
$$

существуют точка $z_{\varepsilon}^{*} \in x^{*}+2 \sqrt{\varepsilon} B_{*}$, субградиент $\xi_{\varepsilon} \in \partial_{\mathrm{F}} f_{\varepsilon}\left(z_{\varepsilon}^{*}\right)$, где $f_{\varepsilon}\left(z^{*}\right):=$ $f\left(y_{\varepsilon}\right)+\left\langle z^{*}, y_{\varepsilon}\right\rangle$, и функция $\varphi$ такие, что

$$
\left\|\zeta-\xi_{\varepsilon}\right\|<\varphi(\varepsilon)
$$

(функция $\varphi$ - та же, что в теореме 4.1).

Пусть $i: \mathbb{X} \rightarrow \mathbb{X}^{* *}$ есть инъекция $\mathbb{X}$ в $\mathbb{X}^{* *}$ такая, что

$$
\left\langle i(y), y^{*}\right\rangle=\left\langle y^{*}, y\right\rangle \quad \forall y \in \mathbb{X}, y^{*} \in \mathbb{X}^{*} .
$$

Легко видеть, что $\xi_{\varepsilon}=i\left(y_{\varepsilon}\right)$, и мы получаем, что для всех малых положительных $\varepsilon$ и $\varepsilon^{\prime}$

$$
\left\|y_{\varepsilon}-y_{\varepsilon^{\prime}}\right\|=\left\|i\left(y_{\varepsilon}\right)-i\left(y_{\varepsilon^{\prime}}\right)\right\|=\left\|\xi_{\varepsilon}-\xi_{\varepsilon^{\prime}}\right\|<\varphi(\varepsilon)+\varphi\left(\varepsilon^{\prime}\right) .
$$

Это означает, что существует предел $y_{0}=\lim _{\varepsilon \downarrow 0} y_{\varepsilon}$ и что $\zeta=i\left(y_{0}\right)$. 
Поскольку $f$ полунепрерывна снизу, $S$ замкнуто и $y_{\varepsilon}$ есть произвольная точка $\varepsilon$-минимума, мы получаем, что $y_{0}$ - это единственная точка минимума функции (48) на $S$. Теорема доказана.

Вариационный принцип Стегалла следует непосредственно из предыдущей теоремы.

СледСтвиЕ 4.2. Пусть $S$ является замкнутым подмножеством банахова пространства $\mathbb{X} u \mathbb{X}^{*}$ гладко. Тогда существует всюду плотное в $\mathbb{X}^{*}$ множество $D$ такое, что для любого $x^{*} \in D$ найдутся точка $y_{0}$ и функиия $\varphi: \mathbb{R}_{+} \rightarrow \mathbb{R}_{+}(\varphi(\varepsilon) \downarrow 0$ при $\varepsilon \downarrow 0)$ такие, что $y_{0}-$ единственная точка минимума функиии (48) на $S$ и для любых $\varepsilon>0$ и $y_{\varepsilon} \in S$, удовлетворяющих $f\left(y_{\varepsilon}\right)+\left\langle x^{*}, y_{\varepsilon}\right\rangle<h\left(x^{*}\right)+\varepsilon$, выполнено неравенство

$$
\left\|y_{\varepsilon}-y_{0}\right\| \leqslant \varphi(\varepsilon)
$$

ДокАЗАТЕЛЬСтво. Поскольку множество $S$ ограничено, мы получаем, что функция $h\left(x^{*}\right)$ липшицева и поэтому ее субдифференциалы непусты на некотором всюду плотном подмножестве $D \subset \mathbb{X}^{*}$.

Выберем $x^{*} \in D$, тогда из теоремы 4.3 следует существование единственной точки минимума $y_{0}$ и функции $\varphi$, а неравенство (50) следует из (49). Следствие доказано.

\section{3. Ближайшие точки для замкнутых подмножеств гильбертова}

пространства. Рассмотрим замкнутое множество $S$ в гильбертовом пространстве $\mathbb{H}$. Для точки $x \notin S$ точка $z \in S$ называется ближайшей или проекцией на $S$, если

$$
\|x-z\|=\inf _{y \in S}\|x-y\| .
$$

Конечно, существование такой ближайшей к $x$ точки для замкнутого множества в гильбертовом пространстве не гарантировано.

Здесь мы демонстрируем применение теоремы 4.1 для доказательства классического результата [46] о существовании единственной проекции на замкнутое подмножество гильбертова пространства для почти всех точек $x$.

Теорема 4.4. Пусть $S \subset \mathbb{H}$ - замкнутое подмножество гильбертова пространства. Тогда существует множество D, всюду плотное в $\mathbb{H}$, такое, что для любой точки $x \in D$ существует единственная ближайшая точка в $S$.

ДокАЗАтеЛЬСтво. Рассмотрим функцию

$$
h(x):=\inf _{y \in S}\|x-y\|^{2} .
$$

Заметим, что $h(x)=d_{S}^{2}(x)$, где $d_{S}-$ функция расстояния до множества $S$.

Функция $h$ локально липшицева, и ее субдифференциал непуст на множестве $D$, всюду плотном в $\mathbb{H}$. Пусть $\zeta \in \partial_{\mathrm{F}} h(x)$ для произвольного $x \in D$; зафиксируем произвольные $\varepsilon>0$ и $y_{\varepsilon} \in S$ такие, что

$$
\left\|x-y_{\varepsilon}\right\|^{2}<d_{S}^{2}(x)+\varepsilon
$$


Заметим, что $y_{\varepsilon}$ есть точка $\varepsilon$-минимума в (51). По теореме 4.1 найдется точка $z_{\varepsilon} \in x+2 \sqrt{\varepsilon} B$ такая, что $\left\|z_{\varepsilon}-y_{\varepsilon}\right\|^{2}<d_{S}^{2}(x)+O(\sqrt{\varepsilon})$ и

$$
\left\|\zeta-2\left(z_{\varepsilon}-y_{\varepsilon}\right)\right\|<\varphi(\varepsilon)
$$

где $2\left(z_{\varepsilon}-y_{\varepsilon}\right)$ есть субградиент функции $z \mapsto\left\|z-y_{\varepsilon}\right\|^{2}$ в точке $z_{\varepsilon}$ и $\varphi(\varepsilon) \downarrow 0$, когда $\varepsilon \downarrow 0$.

Эти соотношения означают, что $y_{\varepsilon}$ сходятся к вектору $x-\zeta / 2$, который и представляет собой ближайшую в $S$ точку для $x$.

Поскольку $y_{\varepsilon}$ есть произвольная точка $\varepsilon$-минимума, получаем, что ближайшая точка единственна. Теорема доказана.

\section{5. Суперградиенты полунепрерывных сверху замыканий полунепрерывных снизу функций}

Рассмотрим полунепрерывное сверху замыкание (3) полунепрерывной снизу функции $f: \mathbb{X} \rightarrow(-\infty,+\infty]$ :

$$
\tilde{f}(x)=(\operatorname{usc} f)(x) .
$$

Ниже мы покажем, что суперградиент $\tilde{f}$ можно приблизить выпуклыми комбинациями субградиентов функции $f$. Аналогичный результат о связи суперградиентов непрерывных функций с их субградиентами был получен в [15] с использованием обобщенной формулы конечных приращений.

Заметим, что этот результат для полунепрерывных снизу функций также связан с "леммой из анализа" полученной Э. Н. Бэрроном и Р. Дженсеном в [4], и может быть использован при сравнении полунепрерывных снизу нижних и верхних решений уравнения Гамильтона-Якоби в случае выпуклого гамильтониана [3]-[5].

Здесь мы также используем этот результат, чтобы получить необходимые условия для точки максимума или точки $\varepsilon$-максимума полунепрерывной снизу функции.

ТеОрема 5.1. Пусть $\mathbb{X}$ - гладкое банахово пространство, функиия $f: \mathbb{X} \rightarrow$ $(-\infty,+\infty]$ полунепрерьвна снизу и выполняется любое из следующих условий:

а) пространство $\mathbb{X}$ рефлексивно;

b) функиия $f$ локально липшищева.

Пусть $\zeta \in \partial^{\mathrm{P}} \tilde{f}(x)$, погда для любых $\delta>0 u \varepsilon>0$ найдутся выпуклые коэфбициенть $\left\{\alpha_{k}\right\}$ и точки $x_{k} \in x+\delta B, k=1, \ldots, K$, такие, что

$$
\zeta \in \sum_{k=1}^{K} \alpha_{k} \partial_{\mathrm{F}} f\left(x_{k}\right)+\varepsilon B .
$$

ДокАЗАтельство. Пусть $\zeta \in \partial^{\mathrm{P}} \tilde{f}(x)$, тогда для некоторой гладкой функции $g$ функция $\tilde{f}-g$ достигает локального максимума в точке $x$ и $\zeta=g^{\prime}(x)$, а это означает, что

$$
\tilde{f}(x)-\tilde{f}(y) \geqslant g(x)-g(y) \quad \text { для любой точки } y \text { вблизи } x \text {. }
$$


Для любого положительного $t$ существует $x_{t} \in x+t^{3} B$ такое, что

$$
f\left(x_{t}\right) \geqslant \tilde{f}(x)-t^{2} .
$$

Выберем произвольный вектор $v \in B$ и заметим, что

$$
f\left(x_{t}+t v\right) \leqslant \tilde{f}\left(x_{t}+t v\right) .
$$

Тогда из (53) получаем, что найдется $\hat{t}$, которое не зависит от $v \in B$, такое, что

$$
f\left(x_{t}\right)-f\left(x_{t}+t v\right)>g(x)-g\left(x_{t}+t v\right)-2 t^{2} \quad \forall t \in(0, \hat{t}) .
$$

Мы используем одномерную формулу конечных приращений из теоремы 2.2 для $r=g(x+t v)-g(x)-2 t^{2}$, точек $x_{t}$ и $y=x_{t}+t v$, чтобы получить существование точки $z$ вблизи $\left[x_{t}, x_{t}+t v\right]$ и субградиента $\xi \in \partial_{\mathrm{F}} f(z)$ таких, что

$$
-\langle\xi, t v\rangle>g(x)-g(x+t v)-2 t^{2} \text {. }
$$

Зафиксируем $\delta>0$ и заметим, что, поскольку $z$ можно выбрать произвольно близкой к $\left[x_{t}, y\right]$, мы можем считать, благодаря (10) и предыдущим соотношениям, что для всех малых положительных $t$

$$
z \in x+\delta B
$$

Пусть выпуклое множество $M$ есть замыкание множества всех выпуклых комбинаций

$$
\sum_{k=1}^{K} \alpha_{k} \zeta_{k}, \quad \text { где } \zeta_{k} \in \partial_{\mathrm{F}} f\left(x_{k}\right), x_{k} \in x+\delta B .
$$

Нетрудно видеть, что из (54) следует, что для всех малых положительных $t$

$$
\sup _{\xi \in M}(-\langle\xi, v\rangle)>\frac{g(x)-g(x+t v)}{t-2 t} .
$$

Беря предел при $t \downarrow 0$ и собрав все члены в левой части неравенства, получаем

$$
\inf _{v \in B} \sup _{\xi \in M}\langle\zeta-\xi, v\rangle \geqslant 0
$$

Заметим, что при выполнении условий а) или b) в предположениях теоремы одно из множеств $B$ или $M$ компактно в соответствующей слабой топологии. Поэтому можно использовать несимметричную теорему о минимаксе [8], чтобы получить из неравенства (55) соотношение $\inf _{\xi \in M}\|\zeta-\xi\|=0$, из которого и следует утверждение теоремы.

Следующий пример показывает, что значения функции $f$ в точках $x_{k}$ не обязаны быть близки к $\tilde{f}(x)$.

ПримеР 5.1. Рассмотрим функцию (28). Нетрудно видеть, что $\zeta=-2$ есть суперградиент usc $f$ в $z:=1 / 2$. Поскольку $\partial_{\mathrm{F}} f(x)=\{\operatorname{sign} x\}$ для всех $x \neq z$, получаем, что существует только одна точка, а именно $z$, такая, что $\zeta \in \partial_{\mathrm{F}} f(z)$. Заметим, что

$$
f(z)<\tilde{f}(z)-\frac{1}{2}
$$


Точки $\varepsilon$-максимума полунепрерывной снизу функции. Субградиенты идеально подходят для того, чтобы характеризовать точки локального минимума полунепрерывных снизу функций: если функция $f$ имеет локальный минимум в $x$, то

$$
0 \in \partial_{\mathrm{F}} f(x) .
$$

Мы покажем, что субградиенты также можно использовать для того, чтобы характеризовать точки максимума или $\varepsilon$-максимума. Напомним, что $x$ называют точкой $\varepsilon$-максимума функции $f$, если

$$
f(x)>\sup _{\mathbb{X}} f-\varepsilon .
$$

Теорема 5.2. Пусть $\mathbb{X}$ - гладкое банахово пространство, функция $f: \mathbb{X} \rightarrow$ $(-\infty,+\infty]$ полунепрерьвна снизу и выполняется любое из следующих условий:

а) пространство $\mathbb{X}$ рефлексивно;

b) функиия $f$ локально липшицева.

Если $x$ есть точка в-максимума функции $f$, то для любых $\lambda>0, \delta>0$ найдутся выпуклье коэфбициенты $\left\{\alpha_{k}\right\}$ и точки $x_{k} \in x+(\lambda+\delta) B, k=$ $1, \ldots, K$, maкue, чmo

$$
0 \in \sum_{k=1}^{K} \alpha_{k} \partial_{\mathrm{F}} f\left(x_{k}\right)+\frac{\varepsilon}{\lambda} B .
$$

ДокАЗАТЕЛЬство. Рассмотрим функцию $\tilde{f}:=\operatorname{usc} f$ и заметим, что $x$ есть точка $\varepsilon$-максимума $\tilde{f}$. Тогда мы имеем

$$
\sup \tilde{f}\left(x+\left(\lambda+\frac{\delta}{3}\right) B\right)-\tilde{f}(x)<\varepsilon .
$$

Применяя многомерную формулу конечных приращений (теорема 2.1) к функции $-\tilde{f}$, множеству $Y:=x+\lambda B$ и $r=-\varepsilon$, мы получаем, что существуют точка $z \in x+(\lambda+2 \delta / 3) B$ и суперградиент $\xi \in \partial^{\mathrm{F}} \tilde{f}(z)$ такие, что

$$
\max _{y \in x+\lambda B}\langle\xi, y-x\rangle<\varepsilon .
$$

Это означает, что

$$
\|\xi\|<\frac{\varepsilon}{\lambda}
$$

и, применяя для $\xi$ формулу (52) из теоремы 5.1, мы приходим к (57). Теорема доказана.

В случае, когда $x$ есть точка максимума полунепрерывной снизу функции $f$, мы получаем следующее утверждение.

СлЕДСТвИЕ 5.1. Пусть $x$ есть точка максимума полунепрерывной снизу функиии $f$. Тогда для любого $\varepsilon>0$ существуют выпуклье коэффициенmъь $\left\{\alpha_{k}\right\}$ и точки $x_{k} \in x+\varepsilon B, k=1, \ldots, K$, такие, что

$$
0 \in \sum_{k=1}^{K} \alpha_{k} \partial_{\mathrm{F}} f\left(x_{k}\right)+\varepsilon B .
$$




\section{6. Субградиенты пределов и эпи-пределов последовательностей функций}

Рассмотрим последовательность полунепрерывных снизу функций $f_{i}: \mathbb{X} \rightarrow$ $(-\infty,+\infty], i=1,2, \ldots$.

Здесь мы приводим формулы для субградиентов верхнего и нижнего пределов

$$
\limsup _{i \rightarrow \infty} f_{i}(x), \quad \liminf _{i \rightarrow \infty} f_{i}(x)
$$

в терминах субградиентов функций $f_{i}(x)$.

В этом разделе мы также приводим формулы для субградиентов эпи-пределов (или Г-пределов) последовательностей полунепрерывных снизу функций

$$
\begin{aligned}
\text { e- } \limsup _{i \rightarrow \infty} f_{i}(x):=\lim _{\delta \downarrow 0} \limsup _{i \rightarrow \infty} \inf _{x^{\prime} \in x+\delta B} f_{i}\left(x^{\prime}\right) \\
\text { e- } \liminf _{i \rightarrow \infty} f_{i}(x):=\lim _{\delta \downarrow 0} \liminf _{i \rightarrow \infty} \inf _{x^{\prime} \in x+\delta B} f_{i}\left(x^{\prime}\right) .
\end{aligned}
$$

Следует отметить, что концепция эпи- (или Г-) сходимости возникает естественным образом при изучении параметрических задач оптимизации [1], [42], непрямых методов вариационного исчисления [21] и обобщенных решений уравнений в частных производных первого порядка [3].

Формулы для субградиентов таких пределов могут быть получены с использованием формул для субградиентов нижних и верхних огибающих из предыдущих разделов, а также следующих представлений пределов в терминах огибающих:

$$
\begin{aligned}
\limsup _{i \rightarrow \infty} f_{i}(x) & =\inf _{n \in \mathbb{N}} \sup _{i \geqslant n} f_{i}(x), \\
\liminf _{i \rightarrow \infty} f_{i}(x) & =\sup _{n \in \mathbb{N}} \inf _{i \geqslant n} f_{i}(x), \\
\text { e- } \limsup _{i \rightarrow \infty} f_{i}(x) & =\sup _{\delta>0} \inf _{n \in \mathbb{N}} \sup _{i \geqslant n} \inf _{x^{\prime} \in x+\delta B} f_{i}\left(x^{\prime}\right), \\
\text { e- } \liminf _{i \rightarrow \infty} f_{i}(x) & =\sup _{\delta>0} \sup _{n \in \mathbb{N}} \inf _{i \geqslant n} \inf _{x^{\prime} \in x+\delta B} f_{i}\left(x^{\prime}\right) .
\end{aligned}
$$

ПРЕДПОЛОЖЕНИЕ 6.1. Въполняется любое из следующих условий:

а) пространство $\mathbb{X}$ рефлексивно;

b) для любого $x \in \mathbb{X}$ найдутся положительные $L$ u $\delta_{0}$ такие, что для произвольных выпуклых коэффициентов $\left\{\alpha_{k}\right\}$, точек $x_{k} \in x+\delta_{0} B$ и субградиентов $\zeta_{k} \in \partial_{\mathrm{F}} f_{i_{k}}\left(x_{k}\right), k=1, \ldots, K$, имеет место неравенство

$$
\left\|\sum_{k=1}^{K} \alpha_{k} \zeta_{k}\right\| \leqslant L .
$$

Заметим, что условие b) выполняется, если $f_{i}$ удовлетворяют равномерному локальному условию Липшица: для точки $x$ существуют постоянные $L$ и $\delta_{0}$ такие, что для всех $i \in \mathbb{N}$ и $x^{\prime}, x^{\prime \prime} \in x+\delta_{0} B$

$$
\left\|f_{i}\left(x^{\prime}\right)-f_{i}\left(x^{\prime \prime}\right)\right\| \leqslant L\left\|x^{\prime}-x^{\prime \prime}\right\| .
$$


Tеорема 6.1. Пусть $\mathbb{X}$ есть гладкое банахово пространство, $f_{i}: \mathbb{X} \rightarrow$ $(-\infty,+\infty], i \in \mathbb{N}$, есть последовательность полунепрерывных снизу функиий и выполняется предположение 6.1 .

1) Если $\zeta \in \partial_{\mathrm{F}} f(x)$, где $f-$ одна из функиий (59)-(61), то для любых $\varepsilon>0$, $\delta>0$ и натурального числа $n$ существуют выпуклье коэффициенты $\left\{\alpha_{k}\right\}$, натуральные числа $i_{k}>n$ и точки $z_{k} \in x+\delta B, k=1, \ldots, K$, такие, что

$$
\zeta \in \sum_{k=1}^{K} \alpha_{k} \partial_{\mathrm{F}} f_{i_{k}}\left(z_{k}\right)+\varepsilon B .
$$

2) Если $\zeta \in \partial_{\mathrm{F}} f(x)$, где $f(x):=\lim _{i \rightarrow \infty} f_{i}(x)$ и последовательность $f_{i}$ сходится $\kappa f$ в некоторой окрестности $x$, то для любых $\varepsilon>0, \delta>0$ существует $n=n(\varepsilon, \delta)$ такое, что для любого $i>n$ найдется $z \in x+\delta B$, для которого выполняется включение

$$
\zeta \in \partial_{\mathrm{F}} f_{i}(z)+\varepsilon B .
$$

ДокАЗАтЕльство. Мы начнем с доказательства утверждения 1) для предельной функции $f(x)$, определенной в (60). Остальные случаи могут быть доказаны по такой же схеме, и мы оставляем их доказательство читателям.

Пусть $\zeta \in \partial_{\mathrm{F}} f(x)$, где $f$ есть функция (60). Тогда существуют $C^{1}$-гладкая функция $g$ и положительное $\delta_{0}$ такие, что $\zeta=g^{\prime}(x)$ и

$$
f(y)-f(x) \geqslant g(y)-g(x) \quad \forall y \in x+\delta_{0} B .
$$

Выберем произвольные $\varepsilon>0, \delta \in\left(0, \delta_{0}\right)$ и определим $t \in(0, \delta / 4)$ такое, что для любого $v \in B$

$$
g(x+t v)-g(x) \geqslant\left\langle g^{\prime}(x), t v\right\rangle-\frac{1}{5} \varepsilon t .
$$

Определим функцию

$$
F_{1}\left(\delta^{\prime}, x\right):=\inf _{n \in \mathbb{N}} \sup _{i \geqslant n} \inf _{x^{\prime} \in x+\delta^{\prime} B} f_{i}\left(x^{\prime}\right) .
$$

Зафиксируем $v \in B$, тогда найдется положительное $\tilde{\delta}=\tilde{\delta}(\varepsilon, t, v)$ такое, что $\tilde{\delta}<\delta / 2$ и для произвольного $\delta^{\prime}<\tilde{\delta}$ выполнено неравенство $F_{1}\left(\delta^{\prime}, x+t v\right)>$ $f(x+t v)-\varepsilon t / 5$. Поскольку $F_{1}\left(\delta^{\prime}, x\right) \leqslant f(x)$, мы получаем из предыдущих неравенств оценку

$$
F_{1}\left(\delta^{\prime}, x+t v\right)-F_{1}\left(\delta^{\prime}, x\right) \geqslant\langle\zeta, t v\rangle-\frac{2}{5} \varepsilon t .
$$

Зафиксируем $\delta^{\prime}$ и рассмотрим функцию

$$
F_{2}(n, x):=\sup _{i \geqslant n} \inf _{x^{\prime} \in x+\delta^{\prime} B} f_{i}\left(x^{\prime}\right) .
$$

Существует $\tilde{n}=\tilde{n}\left(x, \delta^{\prime}\right)$ такое, что для всех $n>\tilde{n}$ выполнено неравенство $F_{2}(n, x) \leqslant F_{1}\left(\delta^{\prime}, x\right)+\varepsilon t / 5$. Поскольку $F_{2}(n, x+t v) \geqslant F_{1}\left(\delta^{\prime}, x+t v\right)$, мы имеем

$$
F_{2}(n, x+t v)-F_{2}(n, x) \geqslant\langle\zeta, t v\rangle-\frac{3}{5} \varepsilon t
$$


Зафиксируем такое $n$ и рассмотрим функцию

$$
F_{3}(i, x):=\inf _{x^{\prime} \in x+\delta^{\prime} B} f_{i}\left(x^{\prime}\right) .
$$

Существует $i \geqslant n$ такое, что выполнено неравенство $F_{3}(i, x+t v)>F_{2}(n, x+t v)-$ $\varepsilon t / 5$. Зафиксируем это $i$; получаем

$$
F_{3}(i, x+t v)-F_{3}(i, x) \geqslant\langle\zeta, t v\rangle-\frac{4}{5} \varepsilon t .
$$

Наконец, выберем $x^{\prime} \in x+\delta^{\prime} B$ такое, что $f_{i}\left(x^{\prime}\right) \leqslant F_{3}(i, x)+\varepsilon t / 5$, и получим следующее неравенство:

$$
f_{i}(y)-f_{i}(x)-\langle\zeta, y-x\rangle>-\varepsilon t, \quad \text { где } y=x+t v .
$$

Теперь мы применяем одномерную формулу конечных приращений, чтобы установить существование точки $z \in x+\delta B$ и субградиента $\xi \in \partial_{\mathrm{F}} f_{i}(z)$ таких, что

$$
\langle\xi-\zeta, t v\rangle>-\varepsilon t
$$

Это неравенство означает, что для любых $n \in \mathbb{N}$ и положительного $\delta$

$$
\inf _{v \in B} \sup _{\xi \in M(n, \delta)}\langle\xi-\zeta, v\rangle>-\varepsilon,
$$

где выпуклое множество $M(n, \delta)$ является замыканием множества всех конечных выпуклых комбинаций вида $\sum_{k=1}^{K} \alpha_{k} \xi_{k}$, где

$$
\xi_{k} \in \partial_{\mathrm{F}} f_{i_{k}}\left(z_{k}\right), \quad i_{k}>n, \quad z_{k} \in x+\delta B, \quad k=1, \ldots, K .
$$

Заметим, что в $(65)$ в соответствии с предположением 6.1 одно из множеств $B$ или $M(n, \delta)$ компактно в соответствующей слабой топологии. Применяя теорему о минимаксе из [8], мы получаем

$$
\inf _{\xi \in M(n, \delta)}\|\xi-\zeta\|<\varepsilon
$$

откуда следует (62).

Чтобы доказать утверждение 2) теоремы, предположим, что $\zeta \in \partial_{\mathrm{F}} f(x)$, и рассмотрим $C^{1}$-гладкую функцию $g: \mathbb{X} \rightarrow \mathbb{R}$ такую, что $\zeta=g^{\prime}(x)$ и (64) справедливо для некоторого $\delta_{0}>0$. Без ограничения общности можно полагать, что $f_{i}$ сходится к $f$ равномерно на $x+\delta_{0} B$.

Выберем произвольные $\varepsilon>0, \delta \in\left(0, \delta_{0}\right)$, тогда из дифференцируемости $g$ и неравенства (64) следует, что существует положительное $t<\delta / 2$ такое, что

$$
f(y)-f(x) \geqslant\left\langle g^{\prime}(x), y-x\right\rangle-\frac{1}{2} \varepsilon t \quad \forall y \in x+t B .
$$

Благодаря равномерной сходимости $f_{i}$, мы получаем существование $n=n(\varepsilon, t)$ такого, что для всех $i>n$

$$
\left|f_{i}(y)-f(y)\right|<\frac{1}{4} \varepsilon t \quad \forall y \in x+\delta_{0} B
$$


Эти неравенства влекут

$$
f_{i}(y)-f_{i}(x)>\langle\zeta, t v\rangle-\varepsilon t \quad \forall y \in x+t B, \quad i>n .
$$

Мы применяем многомерный вариант формулы конечных приращений (теорема 2.1), чтобы получить существование точки $z \in x+2 t B$ и субградиента $\xi \in \partial_{\mathrm{F}} f_{i}(z)$ таких, что

$$
\min _{y \in x+t B}\langle\xi-\zeta, y-x\rangle>-\varepsilon t
$$

что влечет (63).

Отметим, что этот результат в случае дифференцируемости функций $f_{i}$ означает, что

$$
\zeta=\lim _{i \rightarrow \infty} f_{i}^{\prime}\left(z_{i}\right)
$$

для некоторой последовательности $z_{i}$, сходящейся к $x$. Теорема доказана.

Из этой теоремы следует, что в случае равномерной сходимости последовательности $f_{i}$ к $f$ субградиенты $f$ приближаются субградиентами $f_{i}$ для достаточно больших $i$. Однако субградиенты пределов (59)-(61) в общем случае приближаются выпуклыми комбинациями субградиентов $f_{i}$.

Авторы признательны Ф. Кларку, Т. Рокафеллару и Дж. Чжу за полезные и стимулирующие обсуждения.

\section{Список литературы}

[1] H. Attouch, Variational convergence of functions and operators, Appl. Math. Ser., Pitman, Boston, MA, 1984, xiv+423 pp.

[2] W. Ballmann, M. Gromov, V. Schroeder, Manifolds of nonpositive curvature, Progr. Math., 61, Birkhäuser, Boston, MA, 1985, vi+263 pp.

[3] M. Bardi, I. Capuzzo-Dolcetta, Optimal control and viscosity solutions of HamiltonJacobi-Bellman equations, Systems Control Found. Appl., Birkhäuser, Boston, MA, 1997, xviii+570 pp.

[4] E. N. Barron, R. Jensen, "Semicontinuous viscosity solutions for Hamilton-Jacobi equations with convex Hamiltonians", Comm. Partial Differential Equations, 15:12 (1990), 1713-1742.

[5] E. N. Barron, R. Jensen, "Optimal control and semicontinuous viscosity solutions", Proc. Amer. Math. Soc., 113:2 (1991), 397-402.

[6] J. M. Borwein, Q. J. Zhu, "A survey of subdifferential calculus with applications", Nonlinear Anal., 38:6, Ser. A: Theory Methods (1999), 687-773.

[7] J. M. Borwein, Q. J. Zhu, Techniques of variational analysis, CMS Books Math. / Ouvrages Math. SMC, 20, Springer-Verlag, New York, 2005, vi+362 pp.

[8] J. M. Borwein, D. Zhuang, "On Fan's minimax theorem", Math. Program., 34:2 (1986), 232-234.

[9] A. Brønsted, R. T. Rockafellar, "On the subdifferentiability of convex functions", Proc. Amer. Math. Soc., 16:4 (1965), 605-611.

[10] Ф. Кларк, Оптимизация и гладкий анализ, Наука, М., 1988; пер. с англ.: F. H. Clarke, Optimization and nonsmooth analysis, Canad. Math. Soc. Ser. Monogr. Adv. Texts, Wiley, New York, 1983.

[11] F.H. Clarke, Methods of dynamic and nonsmooth optimization, CBMS-NSF Regional Conf. Ser. in Appl. Math., 57, SIAM, Philadelphia, PA, 1989, vi+90 pp. 
[12] Ф. Кларк, Ю. С. Ледяев, "Новые формулы конечных приращений”, Докл. РАН, 331:3 (1993), 275-277; англ. пер.: F. H. Clarke, Yu. S. Ledyaev, "New finiteincrement formulas", Russian Acad. Sci. Dokl. Math., 48:1 (1994), 75-79.

[13] F. Clarke, Yu. S. Ledyaev, "Mean value inequalities in Hilbert space", Trans. Amer. Math. Soc., 344:1 (1994), 307-324.

[14] F.H. Clarke, Yu. S. Ledyaev, "Mean value inequalities", Proc. Amer. Math. Soc., 122:4 (1994), 1075-1083.

[15] F. H. Clarke, Yu. S. Ledyaev, R. J. Stern, "Complements, approximations, smoothings and invariance properties", J. Convex Anal., 4:2 (1997), 189-219; http://www. emis.de/journals/JCA/vol.4_no.2/1.html.

[16] F.H. Clarke, Yu. S. Ledyaev, P. R. Wolenski, "Proximal analysis and minimization principles", J. Math. Anal. Appl., 196:2 (1995), 722-735.

[17] F. H. Clarke, Yu. S. Ledyaev, R. J. Stern, P. R. Wolenski, Nonsmooth analysis and control theory, Grad. Texts in Math., 178, Springer-Verlag, New York, 1998, xiv+276 pp.

[18] J. M. Danskin, "The theory of max-min with applications", SIAM J. Appl. Math., 14 (1966), 641-664.

[19] Д. М. Данскин, Теория максимина и ее приложения $к$ задачам распределения вооружения, Сов. радио, М., 1970, 200 с.; пер. с англ.: J. M. Danskin, The theory of max-min and its application to weapons allocation problems, Econometrics and Operations Research, V, Springer-Verlag, New York, 1967, ix+126 pp.

[20] Л. Данцер, Б. Грюнбаум, В. Кли, Теорема Хелли и ее примененил, Мир, М., 1968, 160 с.; пер. с англ.: L. Danzer, B. Grünbaum, V. Klee, "Helly's theorem and its relatives", Convexity, Proc. Sympos. Pure Math., VII, Amer. Math. Soc., Providence, RI, 1963, 101-180.

[21] E. De Giorgi, G. Del Maso, "Г-convergence and calculus of variations", Mathematical theories of optimization (Genova, 1981), Lecture Notes in Math., 979, Springer, Berlin, 1983, 121-143.

[22] B. V. Dekster, "The Jung theorem in metric spaces of curvature bounded above", Proc. Amer. Math. Soc., 125:8 (1997), 2425-2433.

[23] В.Ф. Демьянов, "К решению некоторых минимаксных задач. I", Кибернетика, 2:6 (1966), 58-66; англ. пер.: V.F. Dem'yanov, "On the solution of several minimax problems. I", Cybernetics, 2 (1966), 47-53; II: Кибернетика, 3:3 (1967), 62-66; англ. пер.: II: Cybernetics, 3 (1967), 51-54.

[24] В.Ф. Демьянов, Минимакс: дифферениируемость по направлениям, Изд-во Ленинград. ун-та, Л., 1974, 112 с.

[25] В.Ф. Демьянов, В.Н. Малоземов, Введение в минимакс, Наука, М., 1972, 368 с.; англ. пер.: V.F. Dem'yanov, V. N. Malozemov, Introduction to minimax, Halsted Press [Wiley], New York-Toronto, ON, 1974, vii+307 pp.

[26] R. Deville, "Stability of subdifferentials of nonconvex functions in Banach spaces", Set-Valued Anal., 2:1-2 (1994), 141-157.

[27] R. Deville, "Smooth variational principles and non-smooth analysis in Banach spaces", Nonlinear analysis, differential equations and control (Montreal, QC, 1998), NATO Sci. Ser. C Math. Phys. Sci., 528, Kluwer Acad. Publ., Dordrecht, 1999, 369-405.

[28] R. Deville, G. Godefroy, V. Zizler, "A smooth variational principle with applications to Hamilton-Jacobi equations in infinite dimensions", J. Funct. Anal., 111:1 (1993), 197-212.

[29] I. Ekeland, G. Lebourg, "Generic Fréchet-differentiability and perturbed optimization problems in Banach spaces", Trans. Amer. Math. Soc., 224:2 (1976), 193-216 (1977).

[30] I. Ekeland, "Nonconvex minimization problems", Bull. Amer. Math. Soc. (N. S. ), 1:3 (1979), 443-474. 
[31] Yu.S. Ledyaev, "On generic existence and uniqueness in nonconvex optimal control problems", Set-Valued Anal., 12:1-2 (2004), 147-162.

[32] Yu. S. Ledyaev, J.S. Treiman, Q. J. Zhu, "Helly's intersection theorem on manifolds of nonpositive curvature", J. Convex Anal., 13:3-4 (2006), 785-798.

[33] Yu. S. Ledyaev, Q. J. Zhu, "Implicit multifunction theorems", Set-Valued Anal., 7:3 (1999), 209-238.

[34] Yu. S. Ledyaev, Q. J. Zhu, "Multidirectional mean value inequalities and weak monotonicity", J. London Math. Soc. (2), 71:1 (2005), 187-202.

[35] Yu. S. Ledyaev, Q. J. Zhu, "Nonsmooth analysis on smooth manifolds", Trans. Amer. Math. Soc., 359:8 (2007), 3687-3732.

[36] B. S. Mordukhovich, Variational analysis and generalized differentiation. I. Basic theory, Grundlehren Math. Wiss., 330, Springer-Verlag, Berlin, 2006, xxii+579 pp.

[37] B. S. Mordukhovich, Variational analysis and generalized differentiation. II. Applications, Grundlehren Math. Wiss., 331, Springer-Verlag, Berlin, 2005, xxii+610 pp.

[38] R. R. Phelps, Convex functions, monotone operators and differentiability, 2nd ed., Lecture Notes in Math., 1364, Springer-Verlag, Berlin, 1993, xii+117 pp.

[39] Б. Н. Пшеничный, “Двойственный метод в экстремальных задачах, I", Кибернетика, 1965, № 3, 89-95; англ. пер.: B. N. Pshenichnyi, "Dual method in extremum problems. I", Cybernetics, 1:3 (1965), 91-99; II: Кибернетика, 1965, № 4, 64-69; англ. пер.: II: Cybernetics, 1:4 (1965), 72-79.

[40] Б. Н. Пшеничный, Необходимые условия экстремума, Наука, М., 1969, 151 с.; англ. пер.: В.N. Pshenichnyi, Necessary conditions for an extremum, Pure and Applied Mathematics, 4, Marcel Dekker, New York, 1971, xviii+230 pp.

[41] Р. Т. Рокафеллар, Выпуклый анализ, Мир, М., 1973, 501 с.; пер. с англ.: R. T. Rockafellar, Convex analysis, Princeton Math. Ser., 28, Princeton Univ. Press, Princeton, NJ, 1970, xviii+451 pp.

[42] R. T. Rockafellar, R. J.-B. Wets, Variational analysis, Grundlehren Math. Wiss., 317, Springer-Verlag, Berlin, 1998, xiv+733 pp.

[43] W. Schirotzek, Nonsmooth analysis, Universitext, Springer, Berlin, 2007, xii+373 pp.

[44] В.Н. Соловьев, “Двойственность невыпуклых экстремальных задач”, Докл. АН CCCP, 314:1 (1990), 135-138; англ. пер.: V. N. Solov'ev, "Duality of nonconvex extremal problems", Soviet Math. Dokl., 42:2 (1991), 380-383.

[45] В.Н. Соловьев, "О субдифференциале и производных по направлениям максимума семейства выпуклых функций. II", Изв. РАН. Сер. матем., 65:1 (2001), 107-132; англ. пер.: V. N. Solov'ev, "The subdifferential and the directional derivatives of the maximum of a family of convex functions. II", Izv. Math., 65:1 (2001), 99-121.

[46] C. Stegall, "Optimization of functions on certain subsets of Banach spaces", Math. Ann., 236:2 (1978), 171-176.

[47] Q. J. Zhu, "Clarke-Ledyaev mean value inequalities in smooth Banach spaces", Nonlinear Anal., 32:3 (1998), 315-324.

Ю. С. Ледяев (Yu. S. Ledyaev)

Математический институт им. В. А. Стеклова РАН;

Western Michigan University, Kalamazoo, USA

E-mail: ledyaev@wmich.edu

Дж. C. Триман (J.S. Treiman)

Western Michigan University, Kalamazoo, USA

E-mail: jay.treiman@wmich.edu
Поступила в редакцию 18.01 .2012 